\title{
Cellulose Nanocrystals: Obtaining and Sources of a Promising Bionanomaterial for Advanced Applications
}

\author{
Taiwo Owoyokun 1(D), Catalina M. Pérez Berumen 2,*(D), Antonia Martínez Luévanos ${ }^{3(\mathbb{D})}$, Liliana \\ Cantú $^{2}$ (i), Ana C. Lara Ceniceros 4 (i) \\ 1 Universidad Autónoma de Coahuila, CYTEMA Graduate Program; t.owoyokun@uadec.edu.mx (T.O.); \\ 2 Universidad Autónoma de Coahuila, Departement of Organic Chemistry; catalinaperez@uadec.edu.mx (C.M.P.B.); \\ lcantu@uadec.edu.mx (L.C.); \\ 3 Universidad Autónoma de Coahuila, Departement of Advanced Ceramic Materials; aml15902@uadec.edu.mx (A.M.L.); \\ 4 Universidad Autónoma de Coahuila, Departement of Advanced Materials; ana.lara@ uadec.edu.mx (A.C.L.C.); \\ * Correspondence: catalinaperez@uade.edu.mx;
}

Scopus Author ID 40861636400

Received: 20.11.2020; Revised: 15.12.2020; Accepted: 16.12.2020; Published: 18.12.2020

\begin{abstract}
Cellulose nanomaterials are one of the most relevant scientific-technological discoveries in recent years. Cellulose nanocrystals (CNCs) stand out among them because their extraordinary chemical, mechanical, thermal, and optical properties make them an interesting alternative to manufacture advanced materials from the most abundant biopolymer on Earth. This work presents a critical analysis of the literature published in the last years, emphasizing the various obtaining processes that have emerged in the search for greener methods. A comparative table of the processes used in the extraction of CNCs from various sources (non-edible biomass and agro-industrial wastes) is included, indicating the process's effectiveness and the characteristics and applications of this sustainable advanced bionanomaterial.
\end{abstract}

Keywords: cellulose; nanocellulose crystals; biopolymer; bionanocoposites; nanomaterials.

(C) 2020 by the authors. This article is an open-access article distributed under the terms and conditions of the Creative Commons Attribution (CC BY) license (https://creativecommons.org/licenses/by/4.0/).

\section{Introduction}

Cellulose nanocrystals (CNCs) are spherical rodlike or needle-like highly crystalline structural substances having diameters ranging between 2 to $50 \mathrm{~nm}$ in width and 50 to $500 \mathrm{~nm}$ in length [1]. It is fascinating that this tiny discovery by Rånby continues to bring colossal results in virtually all areas of science and technology seventy years later.

First, it is essential to point out that cellulose is the most abundant renewable biopolymer on Earth, which implies a tremendous potential for exploitation in practically any country or region worldwide. CNCs are the crystalline constituents of cellulose: the amorphous parts are susceptible to acid attack, which results in having highly-crystalline constituents left after acid hydrolysis [2]. The unique properties related to CNCs and their wide variety and universal availability of sources have made this material have diverse and significantly notable industrial applications $[3,4]$. Over the years, these properties have been reported to be inherent in CNCs: nano dimension in size with high specific surface area, a high aspect ratio, high crystallinity, low density, high and exceptional mechanical strength, unique morphology, high thermal stability, non-abrasive nature, non-toxic, renewability, biocompatibility, and biodegradability, as well as its extraordinary bending strength and modulus, low thermal expansion coefficient, high surface reactivity, and low density [5-7]. 
This review pretends to be a concise update on obtaining cellulose nanocrystals, focusing on sources and processes, emphasizing those different from the classical acid hydrolysis. We analyzed the last ten years' publications, summarizing the most significant results on obtaining the CNCs from diverse sources and their characteristics and applications.

\section{Cellulose Nanomaterials}

The concept of cellulose nanomaterials (CNM) is used to frame all those cellulose nanomaterials bearing different shapes, sizes, chemical surface, and properties [8, 9]. CNMs have attracted significant interest from academia and industry due to their unique and potentially useful properties. Generally, it can be stated that the properties of CNMs are based on three factors: i. the cellulose source, ii. the extraction-production method, and iii. the chemical surface. Thus, CNMs are classified into two broad categories: nano-objects: cellulose nanocrystals, cellulose nanofibrils, and bacterial cellulose, and nanostructured cellulose materials: cellulose microfibrils and cellulose nanocomposites $[8,10]$.

It should be noted that over the years, the CNM nomenclature has been inconsistent, where, for example, CNCs have also been referred to as whiskers, needles, or nanocrystalline cellulose. Likewise, cellulose nanofibrils (CNF) are also known as nano fibrillated, cellulose microfibrils, etc. For this reason, in 2011, the Technical Association of the Cellulose and Paper Industry (TAPPI) established a Nanotechnology Division dedicated to establishing standardization of the definitions of cellulose nanomaterials, and in two abbreviations of different main nano cellulose are declared: cellulose nanocrystals (CNC) and cellulose nanofibrils (CNF) $[7,10,11]$.

Cellulose nanocrystals (CNCs) have been through chemical and physical means. The most common process for obtaining of CNCs is through acid hydrolysis [3, 12, 13]. However, different alternatives have been studied: enzyme-assisted, ultrasonic-assisted, microwaveassisted hydrolysis $[14,15]$, mechanochemical process [16], and using neoteric solvents [17, 18], among others.

Cellulose nanofibrils (CNF) are a nanomaterial obtained from cellulose fibers through chemical or enzymatic treatments and high-shear mechanical treatment, or catalyzed oxidation reactions. CNFs are long and flexible fibers with diameters in nanometers and lengths in micrometers [19, 20].

On the other hand, bacterial cellulose (BC) is a nanocellulose produced through bacterial synthesis (for example, Gluconacetobacter xylinus). BC is a biomaterial with great potential for biological implants and cell immobilization/support for tissue regeneration $[6,19$, 21].

The main differences between $\mathrm{CNC}, \mathrm{CNF}$, and $\mathrm{BC}$ are their dimensions and crystallinity. CNFs have both amorphous and crystalline cellulose constituents, while CNCs have mainly a crystalline form. CNFs are spaghetti-like, highly entangled nanofibers networks, while CNCs are rodlike in shape (like rice grains). CNCs are nano-scale materials, while CNFs and BC could be nano- or micro-scale. Among some companies, a common practice is commercializing their nanocellulose products as "micro-celluloses" (even if they are nanocelluloses) to avoid problems related to the technical characteristics of the nanocellulose and the environmental and toxicity concerns. Table 1 summarizes the concepts used in this work for the nanocellulose objects, other terms commonly used, their characteristics, and their most-used process for obtaining. 


\begin{tabular}{|c|c|c|c|}
\hline $\begin{array}{l}\text { Nanocellulose } \\
\text { type }\end{array}$ & Common terms & $\begin{array}{l}\text { Average size \& Crystallization } \\
\text { Index }\end{array}$ & Obtaining process \\
\hline $\begin{array}{l}\text { Cellulose } \\
\text { nanocrystals } \\
\text { CNC }\end{array}$ & $\begin{array}{l}\text { Nanocellulose crystals (NCC), } \\
\text { crystallites, cellulose whiskers, } \\
\text { nanowhiskers, rodlike cellulose, } \\
\text { nanorods, cellulose } \\
\text { microcrystals. }\end{array}$ & 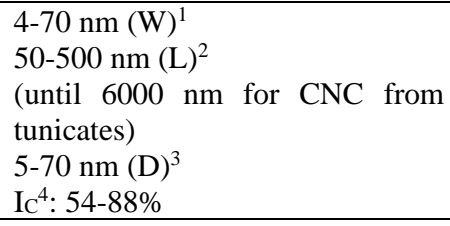 & $\begin{array}{l}\text { Acid hydrolysis, or } \\
\text { combined chemical and } \\
\text { phisical methodologies. }\end{array}$ \\
\hline $\begin{array}{l}\text { Cellulose } \\
\text { nanofibers } \\
\text { CNF }\end{array}$ & 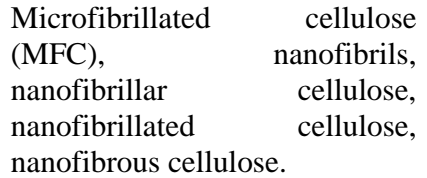 & $\begin{array}{l}29-100 \mathrm{~nm}(\mathrm{~W}) \\
>10,000 \quad \mathrm{~nm} \\
\mathrm{I}_{\mathrm{C}}<50 \%\end{array}$ & $\begin{array}{l}\text { Chemical or enzymatic } \\
\text { pretreatment, followed } \\
\text { by mechanical } \\
\text { treatment. }\end{array}$ \\
\hline $\begin{array}{l}\text { Bacterial } \\
\text { cellulose } \\
\text { BC }\end{array}$ & $\begin{array}{l}\text { Microbial cellulose, bacterial } \\
\text { nanocellulose. }\end{array}$ & $\begin{array}{l}\text { Networks. } \\
\text { Highest IC }\end{array}$ & Bacterial synthesis \\
\hline
\end{tabular}

${ }^{1} \mathrm{~W}$ : width; ${ }^{2} \mathrm{~L}$ : length; ${ }^{3} \mathrm{D}$ :diameter (cross-section); ${ }^{4} \mathrm{I}_{\mathrm{C}}$ : crystallinity index.

\section{Properties and Applications in Research Context}

Research on cellulose nanocrystals has experienced exponential growth for the last ten years. A searching analysis in SciFinder using "nanocellulose crystals" and "extraction" as keywords resulted in the graph shown in Figure 1: there were only 123 publications between 2000 and 2008, and by 2009 there were 63 papers related to nanocellulose crystals; meanwhile, in 2019, were published 1016 articles.

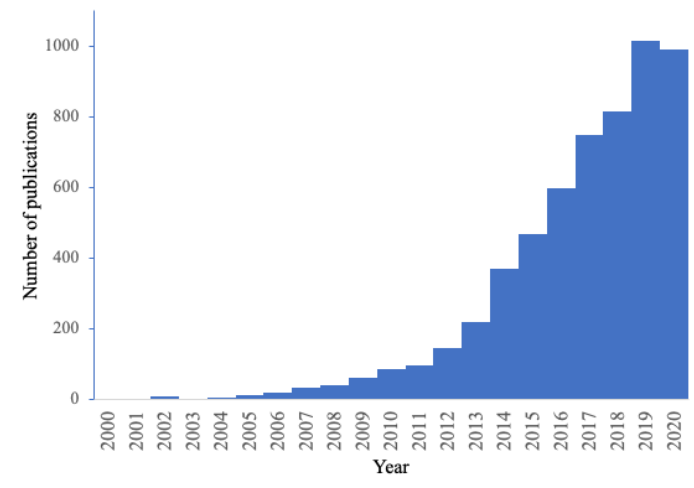

Figure 1. Evolution of the number of publications on CNCs since 2000 (data obtained from SciFinder on October $\left.16^{\text {th }} 2020\right)$.

Analyzing all the publications since 2000, research groups in four countries (China, USA, Canada, and Finland) published almost $80 \%$ of all. The remaining $20 \%$ is from three other countries (France, Sweden, and Switzerland), as shown in figure 2. Research groups from the northern hemisphere are predominant. It is interesting since it signifies an unexploited potential from countries where the vegetal variety and abundance are more prominent.

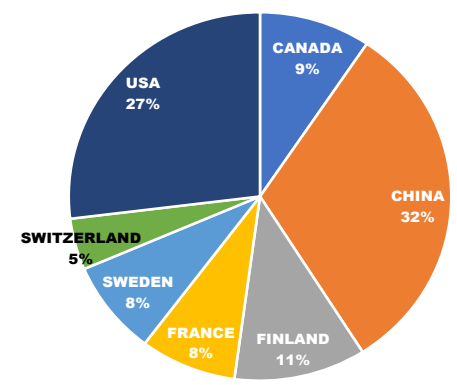

Figure 2. Countries with major contributions on CNCs publications (data obtained from SciFinder on October

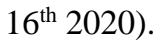


The properties reported for the cellulose nanocrystals implies a remarkably wide range of applications. CNCs have been materials of great industrial and economic worth as pharmaceutical additives and drug delivery excipients [3, 12, 23], in the biomedical area for bone replacement and tooth repair, and as reinforcement in polymer composites and aerogels $[24,25]$. In the alimentary industry, CNCs are used as a food additive [26] and in packaging as antimicrobial [27-29], biodegradable [30], or barrier films [31] and special paper [32, 33]. In optoelectronics, CNCs have been employed as templates for electronic components and composites films with excellent visible light transmittance. They are easily modified and even as flexible displays [34-36]. Nevertheless, without a doubt, the most popular application of $\mathrm{CNCs}$ is as a reinforcement agent for composites for a wide variety of polymer materials [3739]. The extraordinary properties of the CNCs, make them have a wide range of applications: some of these applications are shown in figure 2, where some properties are listed and related to CNCs specific reported use.

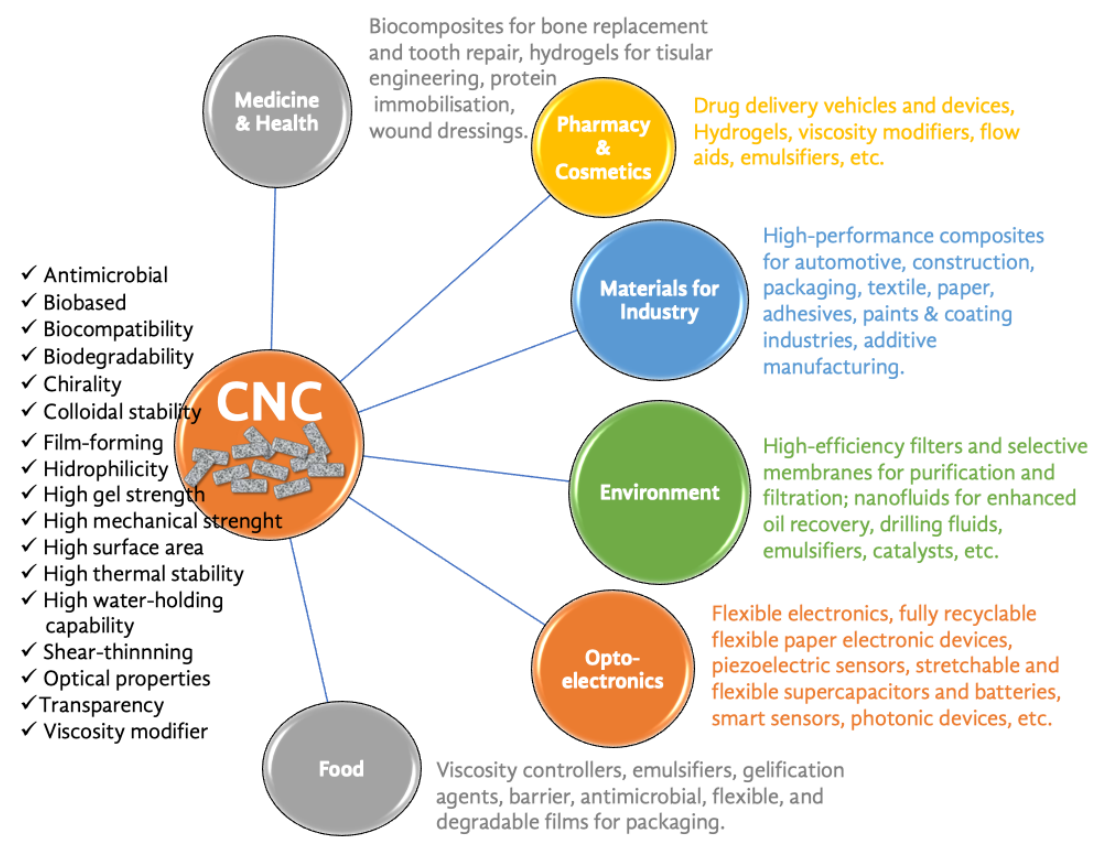

Figure 2. Properties and applications of cellulose nanocrystals.

\section{Different Approaches to Obtain CNCs and from Various Sources}

This section discusses some critical aspects of the classical approach (acid hydrolysis) and innovative methodologies in CNCs obtaining.

\subsection{Acid hydrolysis.}

Acid hydrolysis is the classical methodology for obtaining CNCs, using sulfuric acid ( $64 \% \mathrm{H}_{2} \mathrm{SO}_{4}$, usually between 7 to $10 \mathrm{~mL} / \mathrm{g}$, and heating until $80^{\circ} \mathrm{C}$ for $40-60$ minutes). It has been widely exploited for the production of CNC from diverse sources of cellulose [40].

The most used source of nanocellulose is agricultural residues. For instance, the rice straw has brought about the production of CNCs by acid hydrolysis. After freeze-drying, wellaligned, ultra-fine CNCs were obtained with a high crystallinity ratio and extraordinary structural stability [41]. Also, soy hulls were hydrolyzed with acid for $30 \mathrm{~min}$ has brought about the formation of CNCs that are needle-like with high crystallinity and aspect ratio of 44 . Those CNCs obtained from soy hulls have great potential as reinforcement agents for nanocomposites manufacture [42]. An unusual source, tomato peel, was treated with sulfuric acid and has 
brought to the isolation of CNCs with low yields. However, nanocrystals were reported to be facilely assembled into a fibrous mass with controlled nano-scale diameters. Many applications requiring biodegradable and biocompatible nanofibers can utilize [43].

Other non-alimentary crops have been used in obtaining CNCs. The case for the CNCs isolation from the elephant grass leaves by acid hydrolysis with yields between $12-16 \%$, moderate crystallinity index, and aspect ratios of 30-44. The CNCs have been ascertained to have applications in polymer nanocomposites preparations [44]. The sulfuric acid hydrolysis of kelp cellulose yielded CNCs with verified cellulose I structure and a crystallinity index of $69 \%$ [45]. Another work involves extracting CNCs from delignifed and mercerized sugar palm fibers through acid hydrolysis [46].

To decrease the amounts of sulfuric acid employed in the extraction of CNCs, some research groups have focused on studying diverse methodologies. Among them is using mixtures of different acids or combinations of acids with other solvents or even water but at severe reaction conditions.

For example, under hydrothermal conditions, hydrochloric acid hydrolysis of cellulose raw materials with subsequent neutralization with ammonia has yielded CNCs with a higher yield $(94 \%)$ and crystallinity (89\%), compared with CNCs obtained with typical sulfuric hydrolysis with a $30 \%$ yield and $84 \%$ crystallinity. In both cases, a higher degradation temperature was observed for the $\mathrm{CNCs}$ from hydrochloric acid $\left(\mathrm{T}_{\max }, 363.9^{\circ} \mathrm{C}\right)$ in comparison with those obtained with sulfuric acid $\left(253^{\circ} \mathrm{C}\right)$. In this $\mathrm{HCl}$ hydrolysis, the existence of easily removable ammonium groups through simple heat treatment has enhanced the formation of highly chemically and thermally stable suspensions of the CNCs [47].

In another study, the CNCs obtained through acid hydrolysis of hot water and acetone pretreated spruce bark has a high aspect ratio (>60), high crystallinity, and good thermal stability. The high aspect ratio and good thermal stability have implications for the CNCs obtained from spruce bark to have great potential for use as reinforcement agents in nanocomposites manufacture [48].

Additionally, mixtures with different acids (sulfuric acid and citric/hydrochloric acid) hydrolysis have resulted in sulfonated $\mathrm{CNCs}$ (S-CNCs) and carboxylated CNCs (C-CNCs) nanocrystals, respectively. S-CNCs have an aspect ratio of 59 and $81 \%$ of crystallinity, while the C-CNCs have an aspect ratio of 57 and $83 \%$ crystallinity. Both are needle-like, but S-CNC has higher thermal stability than $\mathrm{C}-\mathrm{CNC}$. Thus, the $\mathrm{CNCs}$ extracted from the Juncus plant possess the potential as reinforcing agents [49]. Acid hydrolysis and subsequent mechanical disintegration process have been used to extract CNCs from alkaline and bleached hemp stalks. CNCs with a high crystallinity index (83\%) have been obtained. They have been applied as nanofillers to produce highly- transparent PVA nanocomposite films [50].

The phosphoric acid hydrolysis has been employed to isolate modified CNCs (phosphorylated CNCs, P-CNCs) from cotton, which is difficult to aggregate and easy to disperse to form stable dispersions in polar solvents like water, dimethyl sulfoxide, and dimethylformamide. The P-CNCs have been reported to have average dimensions and surface charge density. Nanocomposites of an ethylene oxide-epichlorohydrin copolymer and regular $\mathrm{CNCs}$ and P-CNCs were prepared. Composites with P-CNCs exhibit the highest thermal stability, as indicated by thermogravimetric analysis [51].

Acid hydrolysis and subsequent mechanical dispersion have been adopted to isolate CNCs from de-polymerized and bleached marine biomass of brown, red, and green algae through highly thermally stable CNCs high crystalline index (until 99\%) but low aspect ratio 
(15). In comparison with CNCs from other sources, CNCs from algae have shown easy access, higher crystallinity, and better thermal stability. CNCs from algae can be used for increasing the mechanical properties of polymer composites for food packaging [52].

Also, industrial wastes have been the source for obtaining CNCs via acid hydrolysis. For example, CNCs were extracted through sulfuric acid hydrolysis and O-CNCs from ammonium persulfate (APS) one-step oxidation from waste paper fibers. These CNCs showed $22 \%$ and $41 \%$ crystallinity, respectively. In this case, hydrogen peroxide (formed by the APS decomposition) selectively oxidizes the hydroxyl group on the C6 of cellulose into a carboxyl. Successful preparation of CNCs from the fibers obtained from waste paper has created higher economic routes that benefit the transformation of waste papers [43].

The extraction of CNCs from pre-extracted and kraft delignifed loblolly pinewood through sulfuric acid hydrolysis yielded about $70 \%$ (acid concentration and temperature have been reported to be the strongest determinants for the CNC yield). Crystallinity index, surface charge, particle size, and dispersity are relatively uniform, implying consistent quality of CNCs obtained via acid hydrolysis [53].

Expensive economics and operational and environmental hazards associated with acid hydrolysis call for more economical and environmentally friendly means for the production of CNCs.

\subsection{Weak acids methodologies.}

The use of non-conventional technologies, in combination with weak organic acids, have been studied as an alternative for the classic acid hydrolysis. The use of ultrasound combined with organic acids (maleic, oxalic, and citric) has been used successfully to obtain functionalized CNCs, with a yield between 20 and $40 \%$. With a lower energy cost and all the advantages of using mild acids. The carboxylated functionalities on the surface of CNCs make them proper for dispersions in aqueous media [54].

Hydrolysis of bleached eucalyptus kraft pulp (BEKP) fibers through the use of highly recoverable concentrated (50-70 wt\%) oxalic acid have given rise to the production of highly thermally stable and functional CNCs, which proved to be much better than the CNCs obtained through classical acid hydrolysis. The resultant CNCs have high thermal stability $\left(322{ }^{\circ} \mathrm{C}\right)$ and a large aspect ratio with excellent inherent potential for bio-composite applications. High recovery of the organic acids using a conventional crystallization method has made this method uniquely suitable for the sustainable and green production of CNCs [55]. Another simple and effective one-pot esterification and hydrolysis of cellulose from softwood pulp using molten oxalic acid dihydrate have been reported to extract functionalized CNCs with high yield (81\%), surface functionalities, and high thermal stability [56].

Microcrystalline cellulose treatment with citric acid/hydrochloric acid $\left(\mathrm{C}_{6} \mathrm{H}_{8} \mathrm{O}_{7} / \mathrm{HCl}\right)$ has led to the formation of rodlike CNCs subsequently used as cationic dye adsorbent (methylene blue) and flocculant, with a turbidity removal of almost 100\% [57]. Another sustainable and economically feasible use of highly recoverable $\mathrm{FeCl}_{3}$-catalyzed formic acid (FA) to hydrolyze BEKP has resulted in CNCs with a yield higher than $75 \%$ and moderate crystallinity index and thermal stability (around $70 \%$ and $\mathrm{T}_{\max }>260{ }^{\circ} \mathrm{C}$ ). CNCs obtained can be used as building blocks to produce new renewable and sustainable nanomaterials [58].

Relatively fast, highly effective, efficient, energy-intensive, and environmentally friendly hydrolysis using the recyclable phosphotungstic acid (PTA) was used to extract CNCs from cellulose raw materials. This process requires mechanochemical activation but produces 
CNCs in good yields (88\%), a crystallinity index of $80 \%$, and high thermal stability. This technique promises to be an efficient green approach for the preparation of CNCs [59].

An original green methodology employs a subcritical water treatment (at $120^{\circ} \mathrm{C}$ and 20.3 MPa pressure for $60 \mathrm{~min}$ ) of commercial cellulose has given rise to the isolation of rodlike shape CNCs with $22 \%$ yield, having a high crystallinity index (79\%), high thermal stability $\left(300{ }^{\circ} \mathrm{C}\right)$ and high aspect ratio [18]. Another acid-free methodology implies the temperaturecontrolled high-pressure homogenization $(\mathrm{HPH})$, adopted to produce $\mathrm{CNCs}$ having high crystallinity and uniform size. The use of chemicals has been avoided through this technique, representing an efficient and sustainable green approach to CNC production [60].

A combination of TEMPO oxidization pretreatment and mechanical homogenization has produced high yield CNCs with a small width, superthin diameter (3-10 nm) having high crystallinity, carboxylate functional groups, and high surface areas which have potential applications in green nanocomposites ultrafiltration, medicine, and as catalyst supports. The process is less energy-consuming [61]. Also, softwood bleached kraft pulp (SBKP) has been oxidized using an acid-free and dialysis-free process, 2,2,6,6-tetramethylpiperidine-1-oxyl (TEMPO)-mediated system coupled with the sonication process in water for 10 - 120 minutes to produce needle-like TEMPO-CNCs, with a high aspect ratio (50-60). Additionally, TEMPOCNCs have been reported to show higher mass recovery ratios, significantly higher amounts of anionic surface groups, and smaller and more homogeneous widths than the conventional CNCs obtained from acid hydrolysis [62].

On the other hand, ionic liquids have been used as solvent-catalyst pairs for the extraction of CNCs. For instance, 1-ethyl-3-methylimidazolium acetate ([EMIM][OAc]) has been directly used to treat wood directly to extract CNCs. Partially acetylated CNCs with crystallinity index (75\%) and aspect ratio (65) has been obtained [63]. A greener approach that does not produce any hazardous waste employs the highly recoverable ionic liquid $(>90 \%), 1-$ butyl-3-methylimidazolium hydrogen sulfate $\left(\mathrm{BmimHSO}_{4}\right)$, to extract uniform rodlike shape $\mathrm{CNCs}$ having high crystallinity [64]. Another study to optimize the CNCs extraction uses acidic natural deep eutectic solvents (NADES). These NADES were synthesized from choline chloride and oxalic acid at different temperatures and times. A yield of $35 \%$ of CNCs $(80 \%$ crystallinity index) was obtained for a reaction time of $6 \mathrm{~h}$ at $95^{\circ} \mathrm{C}$, opening a novel green route to CNCs [17].

\subsection{Combined methodologies.}

Although the acid-free methods are desirable from the ecological point of view, the real-world results are not the most effective. Consequently, researchers have faced this problem combining the advantages of the hydrolysis (acidic o basic) with the benefits of different activation techniques. In this way, CNCs have been extracted from rice husks through an effective three-step process: alkaline hydrolysis, subsequent bleaching, and final ball milling, a grinding process. The obtained $\mathrm{CNCs}$ have confirmed rice husks as a valuable source to extract valuable nanomaterials for industrial applications [65].

Fabrication of CNCs from the walnut shell through a combination of alkali/acidic hydrolysis in couple with homogenization and ultrasound energy has been achieved. The CNCs have reported having a high yield (92\%), medium crystallinity degree (49\%), and good thermal stability. Walnut shell-derived CNCs can be employed as promising nanocarriers in the food, packaging, delivery nanosystems, and drug delivery sectors [66]. Similarly, CNCs have been 
produced from alkali-treated and bleached apple pomace through acid hydrolysis and ultrasonication treatments, having a 78\% crystallinity [67].

Another method describes the isolation from alkaline treated and bleached Ferula gummosa $(\mathrm{Fg})$ by acid hydrolysis and ultrasonication. The CNCs present co-existence of cellulose I and cellulose II, with high thermal stability and high crystallinity (84\%). The CNCs are highly stable, non-toxic (no cyto-effect), highly biodistributable, and highly effective in metabolic systems such as kidney and blood have been reported. CNCs have proved to have the potential for bioimaging, diagnostic, and other nano-medical applications [68].

A novel methodology concerns the combination of ball milling defibrillation and $\mathrm{SO}_{4}{ }^{2-} / \mathrm{TiO}_{2}$ catalyst-assisted method to extract $\mathrm{CNCs}$ from natural Calotropis gigantea (CGF). This technique is shown to be sustainable and environmentally friendly. $\mathrm{The}^{\mathrm{SO}_{4}}{ }^{2-} / \mathrm{TiO}_{2}$ nanosolid acid exhibited excellent catalytic activity during the ball milling mechanical defibrillation. CNCs with high yield (55\%) and better thermal stability than those obtained from common hydrolysis have been obtained through this technique. A long-anticipated, novel route for the large-scale industrial production of CNCs from biomass and wastes through an environmentally friendly, sustainable green method has been reported [69].

\subsection{Enzymatic hydrolysis.}

Enzymatic hydrolysis is a relatively easy method. It does not require the use of highly corrosive reagents and an enormous quantity of water to obtain CNCs. Also, minimal energy consumption is required, low volumes of water and various commercial enzyme systems allow this procedure to be carried out by non-specialists. However, the yields obtained are generally lower than the purely physical-chemical processes [70].

Enzymatic hydrolysis and mechanical treatment at high pressure have been used to produce CNCs from rice and oat husk-based cellulose. Aerogels have been produced from these CNCs. Rice and oat husks based CNCs and the respective aerogels made from them have been characterized to ascertain their morphology, particle size, functional groups, crystallinity, and thermal properties. The aerogels were reported to have a uniform structure, a high-water absorption capacity, and a large pore size (even more significant than aerogels made from commercial eucalyptus CNCs). CNCs from agricultural biomass and wastes have proven to have promising industrial applications as aerogel absorbers of water in food packaging [71]. A more straightforward and highly economical enzymatic hydrolysis, at a high solid loading (> $15 \% \mathrm{w} / \mathrm{w}$ ), has been incorporated into the biochemical platform process for the production of sugars from sugarcane, has provided a new route to produce CNCs as a high value-added coproduct. The sugarcane bagasse (SCB) based $\mathrm{CNCs}$ have been reported to exhibit better properties than the bleached eucalyptus kraft pulp (BEKP) based CNCs obtained through acid or enzymatic hydrolysis; higher thermal stability, higher crystallinity index, and higher particle diameter uniformity have been attributed to the SCB based CNCs. Much more, this process has a route in which costly sonication process steps necessary to obtain CNC by virtually all methods are eliminated [72].

\subsection{Summary based on the source type.}

The sources for obtaining CNC are worldwide spreading, and practically, in all regions of the planet, exists at least one source of cellulose. However, as previously described, the characteristics of the CNCs depend on the intrinsic properties of the cellulosic raw matter and 
the extraction methodology. With this purpose, table 2 summarizes the most significant articles published in the last years, centered on the extraction of CNC from different sources (based on their nature and alphabetically-listed), specifying the extraction method employed, the yield, the crystallinity index, and the size, as general comparative characteristics of the CNC.

Table 2. Comparative table of publications related to CNCs extraction and applications.

CNCs from biomass (as raw-matter and agro-wastes)

\begin{tabular}{|c|c|c|c|c|c|c|}
\hline \multicolumn{7}{|c|}{ 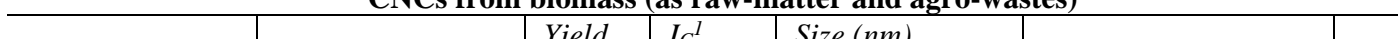 } \\
\hline Sources & Extraction method & $\begin{array}{l}\text { Yield } \\
(\%)\end{array}$ & $\begin{array}{l}I_{C}{ }^{l} \\
(\%) \\
\end{array}$ & $\begin{array}{l}\text { Size }(n m) \\
L^{2}, W^{3}, D^{4}, P S^{5}\end{array}$ & Applications & Ref. \\
\hline \multirow[b]{2}{*}{$\begin{array}{l}\text { Agave tequilana } \\
\text { bagasse }\end{array}$} & \multirow[b]{2}{*}{$\mathrm{H}_{2} \mathrm{SO}_{4}$ hydrolysis } & ns & 71 & $\begin{array}{l}323 \pm 113(\mathrm{~L}) \\
11 \pm 4(\mathrm{D})\end{array}$ & $\begin{array}{l}\text { Reinforcement agent } \\
\text { for nanocomposites }\end{array}$ & [73] \\
\hline & & ns & $89-94$ & $\begin{array}{ll}590 & \text { (L) } \\
100 & \text { (D) } \\
+20(\mathrm{PS}) & \\
\end{array}$ & $\begin{array}{l}\text { Reinforcement agent } \\
\text { for nanocomposites }\end{array}$ & [74] \\
\hline Apple pomace & $\begin{array}{l}\mathrm{H}_{2} \mathrm{SO}_{4} \text { hydrolysis } \\
\& \text { ultrasonication }\end{array}$ & 85 & 78 & $\begin{array}{l}28 \pm 2(\mathrm{~L}) \\
7.9 \pm 1(\mathrm{D})\end{array}$ & $\begin{array}{l}\text { Packaging and } \\
\text { biomedical materials }\end{array}$ & [67] \\
\hline $\begin{array}{l}\text { Asparagus } \\
\text { (Asparagus } \\
\text { officinalis L.) }\end{array}$ & $\mathrm{H}_{2} \mathrm{SO}_{4}$ hydrolysis & ns & $72-77$ & 178-262 (PS) & $\begin{array}{l}\text { Stabilization of } \mathrm{O} / \mathrm{W} \\
\text { Pickering emulsions }\end{array}$ & [75] \\
\hline Bamboo fibers & $\mathrm{H}_{2} \mathrm{SO}_{4}$ hydrolysis & 30 & 87 & $\begin{array}{l}100-130(\mathrm{~L}) \\
5-8(\mathrm{D})\end{array}$ & $\begin{array}{l}\text { Reinforcement agent } \\
\text { for nanocomposites }\end{array}$ & [76] \\
\hline Bamboo shoot & $\mathrm{H}_{2} \mathrm{SO}_{4}$ hydrolysis & 51 & 82 & ns & $\begin{array}{l}\text { Enzyme } \\
\text { immobilization, } \\
\text { tissue engineering }\end{array}$ & [77] \\
\hline $\begin{array}{l}\text { Banana } \\
\text { pseudostem }\end{array}$ & $\begin{array}{l}\mathrm{NaOH} \text { and } \mathrm{H}_{2} \mathrm{SO}_{4} \\
\text { hydrolysis }\end{array}$ & 19 & ns & $\begin{array}{l}1.9-7.2(\mathrm{D}) \\
12-135(\mathrm{~L})\end{array}$ & $\begin{array}{l}\text { Reinforcement agent } \\
\text { for nanocomposites }\end{array}$ & [78] \\
\hline Barley straw & $\mathrm{H}_{2} \mathrm{SO}_{4}$ hydrolysis & 69 & 63 & $\begin{array}{l}160-800(\mathrm{~L}) \\
10-25(\mathrm{~W})\end{array}$ & Reinforcing filler & [79] \\
\hline $\begin{array}{l}\text { Barley straw and } \\
\text { husk }\end{array}$ & $\mathrm{H}_{2} \mathrm{SO}_{4}$ hydrolysis & 12 & ns & $\begin{array}{l}40-270(\mathrm{~L}) \\
5-15(\mathrm{D})\end{array}$ & $\begin{array}{l}\text { Nanoreinforcing } \\
\text { agents in PVA }\end{array}$ & [80] \\
\hline $\begin{array}{l}\text { Cashew tree } \\
\text { pruning }\end{array}$ & $\begin{array}{l}\mathrm{H}_{2} \mathrm{SO}_{4}+\mathrm{HCl} \\
\text { hydrolysis }\end{array}$ & 5.6 & $\mathrm{~ns}$ & $\begin{array}{l}276 \pm 45(\mathrm{~L}) \\
17.5 \pm 4(\mathrm{D})\end{array}$ & $\begin{array}{l}\text { Reinforcing agent in } \\
\text { composite films for } \\
\text { food packaging }\end{array}$ & [81] \\
\hline Coffee husks & $\mathrm{H}_{2} \mathrm{SO}_{4}$ hydrolysis & ns & 92 & $\begin{array}{l}310(\mathrm{~L}) \\
20(\mathrm{~W})\end{array}$ & $\begin{array}{l}\text { Reinforcing agents in } \\
\text { biocomposites }\end{array}$ & [82] \\
\hline \multirow{2}{*}{ Corncob } & \multirow{2}{*}{$\mathrm{H}_{2} \mathrm{SO}_{4}$ hydrolysis } & 41 & 84 & $\begin{array}{l}211 \pm 44(\mathrm{~L}) \\
4 \pm 1(\mathrm{D})\end{array}$ & $\begin{array}{l}\text { Reinforcement agent } \\
\text { in the manufacture of } \\
\text { nanocomposites }\end{array}$ & [83] \\
\hline & & ns & 72 & 131.4 (PS) & $\begin{array}{l}\text { Reinforcement } \\
\text { material in polymer } \\
\text { matrices. }\end{array}$ & [84] \\
\hline Cotton (raw) & $\mathrm{H}_{2} \mathrm{SO}_{4}$ hydrolysis & 77 & 91 & $\begin{array}{l}450 \\
25(W)\end{array}$ & $\begin{array}{l}\text { Aerogels absorber of } \\
\text { water in food } \\
\text { packaging. }\end{array}$ & [85] \\
\hline $\begin{array}{l}\text { Cotton linter } \\
\text { (raw) }\end{array}$ & $\begin{array}{l}\mathrm{H}_{2} \mathrm{SO}_{4} \text { hydrolysis } \\
\& \text { ultrasonication }\end{array}$ & $59-72$ & 82 & $\begin{array}{l}133(\mathrm{~L}) \\
10(\mathrm{~W})\end{array}$ & $\begin{array}{l}\text { Drug delivery, } \\
\text { biomedical, and food } \\
\text { applications. }\end{array}$ & [86] \\
\hline $\begin{array}{l}\text { Crown flower } \\
\text { (Calotropis } \\
\text { gigantean) fiber }\end{array}$ & $\begin{array}{l}\text { Ball milling \& } \\
\mathrm{H}_{2} \mathrm{SO}_{4} \text { hydrolysis } \\
+\quad \text { nanocatalyst- } \\
\text { assisted }\end{array}$ & 55 & 43 & $\begin{array}{l}242.1(\mathrm{~L}) \\
8.8(\mathrm{~W})\end{array}$ & $\begin{array}{l}\text { Production of } \\
\text { advanced } \\
\text { nanomaterials. }\end{array}$ & [69] \\
\hline $\begin{array}{l}\text { Cucumber } \\
\text { (Cucumis } \\
\text { sativus) peels }\end{array}$ & $\mathrm{H}_{2} \mathrm{SO}_{4}$ hydrolysis & 66 & 74 & $\mathrm{~ns}$ & $\begin{array}{l}\text { Bionanocomposites } \\
\text { for food packaging }\end{array}$ & [87] \\
\hline Date palm fibre & $\begin{array}{ll}\mathrm{H}_{2} \mathrm{SO}_{4} & + \\
\mathrm{CH}_{3} \mathrm{CO}_{2} \mathrm{H} & \\
\text { hydrolysis } & \\
\end{array}$ & ns & 84 & $\begin{array}{l}146.5(\mathrm{~L}) \\
8.5(\mathrm{~W})\end{array}$ & $\begin{array}{l}\text { Filler in many } \\
\text { industrial } \\
\text { applications. }\end{array}$ & [88] \\
\hline $\begin{array}{l}\text { Elephant grass } \\
\text { (Pennisetum } \\
\text { purpureum } \\
\text { Sch.) } \\
\end{array}$ & $\mathrm{H}_{2} \mathrm{SO}_{4}$ hydrolysis & $12-16$ & $72-77$ & ns & ns & [44] \\
\hline Garlic skin & $\mathrm{H}_{2} \mathrm{SO}_{4}$ hydrolysis & ns & 63 & 58-96 (PS) & $\begin{array}{l}\text { Reinforcing filler for } \\
\text { bionanocomposites }\end{array}$ & [89] \\
\hline Garlic straw & $\mathrm{H}_{2} \mathrm{SO}_{4}$ hydrolysis & 20 & 69 & $\begin{array}{l}480(\mathrm{~L}) \\
6(\mathrm{~W})\end{array}$ & $\begin{array}{l}\text { Reinforcement agent } \\
\text { for nanocomposites }\end{array}$ & [90] \\
\hline Galbanum & $\mathrm{H}_{2} \mathrm{SO}_{4}$ hydrolysis & ns & 84 & $22.1 \pm 5(\mathrm{D})$ & $\begin{array}{l}\text { Bioimaging and } \\
\text { diagnostics }\end{array}$ & [68] \\
\hline
\end{tabular}




\begin{tabular}{|c|c|c|c|c|c|c|}
\hline $\begin{array}{l}\text { (Ferula } \\
\text { gummosa) }\end{array}$ & & & & & & \\
\hline Grape pomace & $\mathrm{H}_{2} \mathrm{SO}_{4}$ hydrolysis & 70 & 71 & ns & $\begin{array}{l}\text { Starch-based } \\
\text { biocomposites }\end{array}$ & [91] \\
\hline $\begin{array}{l}\text { Green algae } \\
\text { (Cladophora } \\
\text { rupestris) } \\
\end{array}$ & $\mathrm{HBr}$ hydrolysis & $\mathrm{ns}$ & 94 & $20.0 \pm 4(\mathrm{D})$ & $\begin{array}{l}\text { Starch based } \\
\text { biocomposites }\end{array}$ & [92] \\
\hline $\begin{array}{l}\text { Harakeke } \\
\text { (Phormium } \\
\text { tenax) }\end{array}$ & $\mathrm{H}_{2} \mathrm{SO}_{4}$ hydrolysis & 35 & ns & $\begin{array}{l}100-200(\mathrm{~L}) \\
5-10(\mathrm{~W})\end{array}$ & $\begin{array}{l}\text { Reinforcement agent } \\
\text { for nanocomposites }\end{array}$ & [93] \\
\hline Hemp & $\mathrm{H}_{2} \mathrm{SO}_{4}$ hydrolysis & 38 & 84 & $\begin{array}{l}20-120(\mathrm{~L}) \\
1-4.5(\mathrm{D}) \\
\end{array}$ & $\begin{array}{l}\text { Bionanocomposite } \\
\text { films }\end{array}$ & [94] \\
\hline Hemp stalk & $\mathrm{H}_{2} \mathrm{SO}_{4}$ hydrolysis & $\mathrm{ns}$ & 83 & $\begin{array}{l}367 \pm 101 \text { (L) } \\
7.4 \pm 2.2(\mathrm{D})\end{array}$ & $\begin{array}{l}\text { Reinforcements in } \\
\text { PVA polymers }\end{array}$ & {$[50]$} \\
\hline $\begin{array}{l}\text { Japanese hop } \\
\text { (Humulus } \\
\text { japonicus) }\end{array}$ & $\mathrm{H}_{2} \mathrm{SO}_{4}$ hydrolysis & 30 & 70 & 6.48 (D) & $\begin{array}{l}\text { Nanocomposites, } \\
\text { biomedical, and food } \\
\text { applications. }\end{array}$ & [95] \\
\hline \multirow{2}{*}{ Juncus stems } & $\mathrm{H}_{2} \mathrm{SO}_{4}$ hydrolysis & ns & 81 & $\begin{array}{l}431 \pm 94(\mathrm{~L}) \\
7.3 \pm 2(\mathrm{D})\end{array}$ & \multirow{2}{*}{$\begin{array}{l}\text { Nanoreinforcing } \\
\text { agents in polymers }\end{array}$} & \multirow{2}{*}{ [49] } \\
\hline & $\begin{array}{l}\text { Citric acid/HCl } \\
\text { hydrolysis }\end{array}$ & ns & 83 & $6.1 \pm 2.8(\mathrm{D})$ & & \\
\hline Jute fibre & $\begin{array}{l}\mathrm{TEMPO} / \mathrm{NaClO} / \\
\mathrm{NaBr} \text { system }\end{array}$ & 80 & 68 & $\begin{array}{l}100-200(\mathrm{~L}) \\
3-10(\mathrm{~W})\end{array}$ & $\begin{array}{l}\text { Additives in tissue } \\
\text { eng., pharmaceutical } \\
\text { and optical industries }\end{array}$ & [61] \\
\hline $\begin{array}{l}\text { Kapok }(\text { Ceiba } \\
\text { pentandra) fiber }\end{array}$ & $\mathrm{H}_{2} \mathrm{SO}_{4}$ hydrolysis & 32 & 89 & $\begin{array}{l}79.4 \pm 13(\mathrm{~L}) \\
4.2 \pm 3(\mathrm{~W})\end{array}$ & $\begin{array}{l}\text { Self-assembled } \\
\text { membranes }\end{array}$ & [96] \\
\hline $\begin{array}{l}\text { King grass } \\
(\text { Pennisetum } \\
\text { sinese }) \\
\end{array}$ & $\mathrm{H}_{2} \mathrm{SO}_{4}$ hydrolysis & 45 & 77 & $\begin{array}{l}20-30(\mathrm{D}) \\
200-300(\mathrm{~L})\end{array}$ & $\begin{array}{l}\text { Reinforcement agent } \\
\text { in composite } \\
\text { manufacturing }\end{array}$ & [97] \\
\hline $\begin{array}{l}\text { Kudzu } \\
\text { (Pueraria } \\
\text { lobate) root } \\
\text { residue }\end{array}$ & $\begin{array}{l}\text { Phosphoric acid } \\
\text { hydrolysis }\end{array}$ & $\mathrm{ns}$ & 48 & $\begin{array}{l}100-330(\mathrm{~L}) \\
2-6(\mathrm{~W})\end{array}$ & $\begin{array}{l}\text { Nanoreinforcing } \\
\text { agents in polymers }\end{array}$ & [98] \\
\hline $\begin{array}{l}\text { Mengkuang } \\
\text { (Pandanus } \\
\text { odoratissinus) } \\
\text { leaves }\end{array}$ & $\begin{array}{l}\mathrm{NaOH} \text { and } \mathrm{H}_{2} \mathrm{SO}_{4} \\
\text { hydrolysis }\end{array}$ & 28 & 66.7 & $\begin{array}{l}5-80(\mathrm{~L}) \\
5-25(\mathrm{D})\end{array}$ & $\begin{array}{l}\text { Reinforcement agent } \\
\text { in the manufacture of } \\
\text { PVC nanocomposites }\end{array}$ & [99] \\
\hline $\begin{array}{l}\text { Misacanthus } \\
\text { (Miscanthus } \\
\text { giganteus) }\end{array}$ & $\mathrm{H}_{2} \mathrm{SO}_{4}$ hydrolysis & 33 & 90 & $\begin{array}{l}8.5(\mathrm{~W}) \\
2.8(\mathrm{~T})^{6}\end{array}$ & $\begin{array}{l}\text { Nanocomposite in } \\
\text { PVA polymers }\end{array}$ & [100] \\
\hline Oats husks & $\begin{array}{l}\text { Enzymatic } \\
\text { hydrolysis \& } \\
\text { mechanical } \\
\text { treatment at high } \\
\text { pressure }\end{array}$ & ns & 90 & 28.8 (D) & $\begin{array}{l}\text { Aerogel absorber in } \\
\text { food packaging }\end{array}$ & [71] \\
\hline Oil palm trunk & $\mathrm{H}_{2} \mathrm{SO}_{4}$ hydrolysis & ns & 74.81 & $\begin{array}{l}82.8(\mathrm{~L}) \\
3.6(\mathrm{D})\end{array}$ & $\begin{array}{l}\text { Polymer } \\
\text { nanocomposite, food } \\
\text { packaging }\end{array}$ & [101] \\
\hline $\begin{array}{l}\text { Okra } \\
\text { (Abelmoschus } \\
\text { esculentus) } \\
\text { fibers }\end{array}$ & $\begin{array}{l}\text { Microbial } \\
\text { degradation, } \\
\mathrm{H}_{2} \mathrm{SO}_{4} \text {, and } \mathrm{NaOH} \\
\text { hydrolysis, } \\
\text { bleaching } \\
\end{array}$ & 30 & ns & ns & $\begin{array}{l}\text { Production } \\
\text { PVA/CNC } \\
\text { nanocomposites }\end{array}$ & [102] \\
\hline Onion skin & $\mathrm{H}_{2} \mathrm{SO}_{4}$ hydrolysis & 49 & 26 & $2.49(\mathrm{PS})$ & Biocomposite films & [103] \\
\hline $\begin{array}{ll}\text { Passion fruit } \\
\text { peels }\end{array}$ & $\mathrm{H}_{2} \mathrm{SO}_{4}$ hydrolysis & 58 & 78 & 145.4 (PS) & $\begin{array}{ll}\begin{array}{l}\text { Drug } \\
\text { excipients }\end{array} & \text { carrier } \\
\end{array}$ & [104] \\
\hline Pineapple leafs & $\mathrm{H}_{2} \mathrm{SO}_{4}$ hydrolysis & 68 & 73 & $\begin{array}{l}250 \pm 51(\mathrm{~L}) \\
4 \pm 1(\mathrm{D})\end{array}$ & $\begin{array}{l}\text { Reinforcement agent } \\
\text { for nanocomposites }\end{array}$ & [105] \\
\hline $\begin{array}{l}\text { Pistachio } \\
\text { (Pistacia vera) } \\
\text { shells }\end{array}$ & $\mathrm{HCl}$ hydrolysis & 77 & 79 & $68.8 \pm 21(\mathrm{D})$ & $\begin{array}{l}\text { Stabilization of } \mathrm{O} / \mathrm{W} \\
\text { Pickering emulsions. }\end{array}$ & [106] \\
\hline Pea hull & $\mathrm{H}_{2} \mathrm{SO}_{4}$ hydrolysis & $\mathrm{ns}$ & 77 & $\begin{array}{l}81-286(\mathrm{~L}) \\
8-21(\mathrm{D})\end{array}$ & $\begin{array}{l}\text { Food packaging } \\
\text { applications }\end{array}$ & [107] \\
\hline $\begin{array}{l}\text { Peanut (Arachis } \\
\text { hypogaea) shell }\end{array}$ & $\mathrm{H}_{2} \mathrm{SO}_{4}$ hydrolysis & 12 & 74 & $\begin{array}{l}67-172(\mathrm{~L}) \\
5-8(\mathrm{~W})\end{array}$ & $\begin{array}{l}\text { Reinforcement agent } \\
\text { for nanocomposite, } \\
\text { packaging, drug } \\
\text { delivery. }\end{array}$ & [108] \\
\hline Potato peel & $\mathrm{H}_{2} \mathrm{SO}_{4}$ hydrolysis & ns & ns & $100-200$ (PS) & $\begin{array}{l}\text { Reinforcing agent for } \\
\text { the production of }\end{array}$ & [109] \\
\hline
\end{tabular}




\begin{tabular}{|c|c|c|c|c|c|c|}
\hline & & & & & $\begin{array}{l}\text { hydrogel } \\
\text { nanocomposites }\end{array}$ & \\
\hline $\begin{array}{l}\text { Ramie } \\
\text { (Boehmeria } \\
\text { nivea) fiber }\end{array}$ & $\mathrm{H}_{2} \mathrm{SO}_{4}$ hydrolysis & ns & 91 & $\begin{array}{l}145.61 \text { (L) } 6.67 \\
\text { (D) }\end{array}$ & $\begin{array}{l}\text { Reinforcing fillers for } \\
\text { nanocomposites }\end{array}$ & {$[110]$} \\
\hline $\begin{array}{ll}\begin{array}{l}\text { Red } \\
\text { (waste) }\end{array} & \text { algae } \\
\end{array}$ & $\mathrm{H}_{2} \mathrm{SO}_{4}$ hydrolysis & ns & 84 & $\begin{array}{l}285 \pm 36(\mathrm{~L}) \\
5 \pm 2(\mathrm{D})\end{array}$ & $\begin{array}{l}\text { PVA nanocomposite } \\
\text { films }\end{array}$ & [111] \\
\hline Rice husks & $\begin{array}{l}\text { Enzymatic } \\
\text { hydrolysis and } \\
\text { mechanical } \\
\text { treatment at high } \\
\text { pressure }\end{array}$ & ns & 60 & 16.4 (D) & $\begin{array}{l}\text { Aerogels absorber of } \\
\text { water in food } \\
\text { packaging }\end{array}$ & {$[71]$} \\
\hline $\begin{array}{l}\text { Rice husks } \\
\text { (large grain) }\end{array}$ & \multirow{3}{*}{$\begin{array}{l}\mathrm{H}_{2} \mathrm{SO}_{4} \text { hydrolysis } \\
\text { and sonication }\end{array}$} & ns & 77 & $\begin{array}{l}153-779 \text { (L) 20- } \\
48 \text { (D) }\end{array}$ & \multirow{3}{*}{$\begin{array}{l}\text { Polymer } \\
\text { nanocomposites }\end{array}$} & \multirow{3}{*}[112]{} \\
\hline $\begin{array}{l}\text { Rice husks } \\
\text { (medium grain) }\end{array}$ & & ns & 62 & $\begin{array}{l}111-477 \text { (L) 16- } \\
38 \text { (D) }\end{array}$ & & \\
\hline $\begin{array}{l}\text { Rice husks } \\
\text { (small grain) }\end{array}$ & & ns & 53 & $\begin{array}{ll}56-179 & \text { (L) } 12- \\
29 & \text { (D) }\end{array}$ & & \\
\hline \multirow[b]{2}{*}{ Rice straws } & \multirow[b]{2}{*}{$\mathrm{H}_{2} \mathrm{SO}_{4}$ hydrolysis } & 90 & 76 & $5-15(\mathrm{D})$ & \multirow[b]{2}{*}{$\begin{array}{l}\text { Reinforcing agent for } \\
\text { biocomposites }\end{array}$} & {$[13]$} \\
\hline & & 64 & 66 & $\begin{array}{l}130-650(\mathrm{~L}) \\
12-20(\mathrm{~W})\end{array}$ & & {$[79]$} \\
\hline Sago seed shell & $\mathrm{H}_{2} \mathrm{SO}_{4}$ hydrolysis & ns & $69-72$ & $10-50(\mathrm{PS})$ & $\begin{array}{l}\text { Reinforcement } \\
\text { material in polymer } \\
\text { matrices }\end{array}$ & {$[113]$} \\
\hline $\begin{array}{l}\text { Sedge (Carex } \\
\text { meyeriana } \\
\text { Kunth) }\end{array}$ & $\begin{array}{l}\text { TEMPO oxidation } \\
\text { and mechanical } \\
\text { homogenization }\end{array}$ & ns & 51.01 & $\begin{array}{l}175(\mathrm{~L}) \\
33(\mathrm{D})\end{array}$ & $\begin{array}{l}\text { Biosorbent in } \\
\text { wastewater treatment }\end{array}$ & {$[114]$} \\
\hline Sisal fiber & $\mathrm{H}_{2} \mathrm{SO}_{4}$ hydrolysis & ns & 73 & $\begin{array}{l}128 \pm 20(\mathrm{~L}) 9.4 \\
\pm 1.8(\mathrm{D})\end{array}$ & $\begin{array}{l}\text { Nanomaterials in } \\
\text { electronics, optics, } \\
\text { biomedicals }\end{array}$ & {$[115]$} \\
\hline $\begin{array}{l}\text { Small } \\
\text { crownflower } \\
\text { (Calotropis } \\
\text { procera) }\end{array}$ & $\mathrm{H}_{2} \mathrm{SO}_{4}$ hydrolysis & ns & 68.7 & $\begin{array}{l}250(\mathrm{~L}) \\
12(\mathrm{~W})\end{array}$ & $\begin{array}{l}\text { Renewable } \\
\text { nanocomposites }\end{array}$ & {$[116]$} \\
\hline Soy hulls & $\mathrm{H}_{2} \mathrm{SO}_{4}$ hydrolysis & 59 & 74 & $\begin{array}{l}123 \pm 39(\mathrm{~L}) \\
3 \pm 1(\mathrm{D}) \\
\end{array}$ & $\begin{array}{l}\text { Reinforcing agent for } \\
\text { composites }\end{array}$ & [117] \\
\hline \multirow{4}{*}{$\begin{array}{l}\text { Sugarcane } \\
\text { bagasse }\end{array}$} & $\begin{array}{l}\mathrm{KOH} \text { and } \mathrm{H}_{2} \mathrm{SO}_{4} \\
\text { hydrolysis }\end{array}$ & 64 & 73 & $\begin{array}{l}250-480(\mathrm{~L}) 20- \\
60(\mathrm{~W})\end{array}$ & $\begin{array}{l}\text { Reinforcing agent for } \\
\text { composites for } \\
\text { biomedical products }\end{array}$ & [118] \\
\hline & \multirow[b]{2}{*}{$\mathrm{H}_{2} \mathrm{SO}_{4}$ hydrolysis } & 54 & 80 & $\begin{array}{l}14-18 \text { (D) 193- } \\
246(\mathrm{~L})\end{array}$ & $\begin{array}{l}\text { Reinforcing agent for } \\
\text { composites }\end{array}$ & [119] \\
\hline & & $\mathrm{ns}$ & 77 & 38 (D) & $\begin{array}{l}\text { Composite either for } \\
\text { biomedical } \\
\text { applications or for } \\
\text { wastewater treatment }\end{array}$ & {$[120]$} \\
\hline & $\begin{array}{l}\text { Enzymatic } \\
\text { hydrolysis (high } \\
\text { solid loading) }\end{array}$ & 50 & 97 & 20 (D) & $\begin{array}{l}\text { Reinforcement agent } \\
\text { for polymer } \\
\text { composites }\end{array}$ & {$[72]$} \\
\hline $\begin{array}{l}\text { Sunflower seed } \\
\text { hull }\end{array}$ & $\mathrm{H}_{2} \mathrm{SO}_{4}$ hydrolysis & 30 & $\begin{array}{l}\text { High } \\
\text { (ns) }\end{array}$ & $\begin{array}{l}150-300(\mathrm{~L}) 10- \\
30(\mathrm{D})\end{array}$ & $\begin{array}{l}\text { Reinforcement } \\
\text { material in polymer } \\
\text { matrices }\end{array}$ & {$[121]$} \\
\hline Switchgrass & $\mathrm{H}_{2} \mathrm{SO}_{4}$ hydrolysis & ns & 72 & $\begin{array}{l}148 \pm 42(\mathrm{~L}) \\
21 \pm 4(\mathrm{~W}) \\
3 \pm 1(\mathrm{~T}) \\
\end{array}$ & $\begin{array}{l}\text { Reinforcing agent for } \\
\text { composites }\end{array}$ & {$[122]$} \\
\hline Tomato peels & $\mathrm{H}_{2} \mathrm{SO}_{4}$ hydrolysis & 16 & 81 & $\begin{array}{l}135(\mathrm{~L}) \\
7.2(\mathrm{~W}) \\
3.3(\mathrm{~T}) \\
\end{array}$ & $\begin{array}{l}\text { Biodegradable } \\
\text { biocompatible } \\
\text { nanofibers }\end{array}$ & {$[123]$} \\
\hline \multirow[b]{2}{*}{ Tunicate } & $\mathrm{H}_{2} \mathrm{SO}_{4}$ hydrolysis & 30 & 91 & $\begin{array}{l}694 \pm 312(\mathrm{~L}) \\
20 \pm 3(\mathrm{~W}) \\
\end{array}$ & \multirow{2}{*}{$\begin{array}{l}\text { Edible, biodegradable } \\
\text { polymeric films in the } \\
\text { food and } \\
\text { pharmaceutical } \\
\text { industries }\end{array}$} & \multirow[b]{2}{*}[124]{} \\
\hline & $\begin{array}{l}\text { Enzymatic } \\
\text { hydrolysis with } \\
\text { subsequent } \\
\text { ultrasonication } \\
\end{array}$ & 73 & 83 & $17 \pm 2.7(\mathrm{~W})$ & & \\
\hline Vine shoot & $\mathrm{H}_{2} \mathrm{SO}_{4}$ hydrolysis & ns & 82 & $\begin{array}{l}456(\mathrm{~L}) \\
14(\mathrm{D})\end{array}$ & $\begin{array}{l}\text { Reinforcing agent for } \\
\text { nanocomposites }\end{array}$ & {$[125]$} \\
\hline Walnut shell & $\mathrm{H}_{2} \mathrm{SO}_{4}$ hydrolysis & 88 & 60 & $130 \pm 16(\mathrm{D})$ & $\begin{array}{l}\text { Nanocarrier in the } \\
\text { food and drug } \\
\text { delivery }\end{array}$ & {$[66]$} \\
\hline
\end{tabular}




\begin{tabular}{|c|c|c|c|c|c|c|}
\hline Wheat straws & $\mathrm{H}_{2} \mathrm{SO}_{4}$ hydrolysis & 75 & 71 & $\begin{array}{l}120-600(\mathrm{~L}) \\
15-20(\mathrm{~W}) \\
\end{array}$ & $\begin{array}{l}\text { Reinforcing agent for } \\
\text { composites }\end{array}$ & [79] \\
\hline \multicolumn{7}{|c|}{ CNCs from wastes (different from agro-industries) } \\
\hline Sources & Extraction method & $\begin{array}{l}\text { Yield } \\
(\%)\end{array}$ & $\begin{array}{l}I_{C} \\
(\%) \\
\end{array}$ & $\begin{array}{l}\text { Size }(n m) \\
W, L, D, T\end{array}$ & Applications & Ref. \\
\hline Cotton & $\begin{array}{l}\mathrm{NaOH} \text { and } \mathrm{H}_{2} \mathrm{SO}_{4} \\
\text { hydrolysis }\end{array}$ & 45 & ns & $\begin{array}{l}180 \pm 6(\mathrm{~L}) \\
10 \pm 1(\mathrm{~W}) \\
6.5(\mathrm{~T})\end{array}$ & $\begin{array}{l}\text { Biosensors, food } \\
\text { packaging, and drug } \\
\text { delivery applications. }\end{array}$ & [126] \\
\hline $\begin{array}{l}\text { Cotton (from } \\
\text { cloth waste) }\end{array}$ & $\begin{array}{l}\text { Ultrasonic } \\
\text { assisted } \mathrm{HCl} \text { and } \\
\mathrm{H}_{2} \mathrm{SO}_{4} \text { hydrolysis }\end{array}$ & 47 & 56 & $\begin{array}{l}28-470(\mathrm{~L}) \\
3-35(\mathrm{~W})\end{array}$ & $\begin{array}{l}\text { Enhancer for } \\
\text { bionanocomposites }\end{array}$ & [127] \\
\hline $\begin{array}{l}\text { Cotton (from } \\
\text { hospitals waste) }\end{array}$ & $\begin{array}{l}\text { Ultrasonic } \\
\text { assisted } \mathrm{H}_{2} \mathrm{SO}_{4} \\
\text { hydrolysis }\end{array}$ & ns & 81 & $10-50(\mathrm{D})$ & $\begin{array}{l}\text { Biosensors and } \\
\text { nanocomposites }\end{array}$ & [128] \\
\hline $\begin{array}{l}\text { Disposable cup } \\
\text { waste }\end{array}$ & $\begin{array}{ll}\text { Citric } & \text { acid } \\
\text { hydrolysis }\end{array}$ & 95 & $70-94$ & $\begin{array}{l}480(\mathrm{~L}) \\
14(\mathrm{~W})\end{array}$ & ns & [129] \\
\hline $\begin{array}{l}\text { Domestic } \\
\text { wastewater } \\
\text { sludge } \\
\end{array}$ & $\mathrm{H}_{2} \mathrm{SO}_{4}$ hydrolysis & 22 & $62-80$ & $\begin{array}{l}130(\mathrm{~L}) \\
10(\mathrm{~W})\end{array}$ & $\begin{array}{l}\text { Bionanocomposite } \\
\text { films }\end{array}$ & [130] \\
\hline Grass waste & $\mathrm{H}_{2} \mathrm{SO}_{4}$ hydrolysis & $\mathrm{ns}$ & 58 & $\begin{array}{l}100-500(\mathrm{~L}) \\
5-15(\mathrm{D})\end{array}$ & ns & [131] \\
\hline Old newspaper & $\mathrm{H}_{2} \mathrm{SO}_{4}$ hydrolysis & $93-96$ & $34-75$ & $\begin{array}{l}218-317(\mathrm{~L}) \\
2.9-3.2(\mathrm{~W})\end{array}$ & $\mathrm{ns}$ & [132] \\
\hline \multirow[b]{2}{*}{ Paper } & $\mathrm{H}_{2} \mathrm{SO}_{4}$ hydrolysis & 22 & 77 & $\begin{array}{l}200 \pm 25(\mathrm{~L}) \\
8 \pm 2.5(\mathrm{D})\end{array}$ & \multirow[b]{2}{*}{$\begin{array}{l}\text { Reinforcing agent for } \\
\text { polymer composites }\end{array}$} & \multirow[b]{2}{*}{ [43] } \\
\hline & $\begin{array}{l}\text { Ammonium } \\
\text { persulfate (APS) } \\
\text { oxidation }\end{array}$ & 41 & 72 & $\begin{array}{l}130 \pm 15(\mathrm{~L}) \\
4 \pm 2.5(\mathrm{D})\end{array}$ & & \\
\hline Papermill sludge & $\mathrm{H}_{2} \mathrm{SO}_{4}$ hydrolysis & 27 & 93.2 & 130.8 (PS) & $\begin{array}{l}\text { Reinforcing fillers for } \\
\text { composites }\end{array}$ & [133] \\
\hline $\begin{array}{ll}\text { Rejected } & \text { fiber } \\
\text { from } & \text { kraft } \\
\text { pulping } & \\
\end{array}$ & $\mathrm{H}_{2} \mathrm{SO}_{4}$ hydrolysis & 37 & 83 & $10-35(\mathrm{D})$ & $\begin{array}{l}\text { Reinforcing filler for } \\
\text { polymer composites }\end{array}$ & [134] \\
\hline Sawdust & $\mathrm{H}_{2} \mathrm{SO}_{4}$ hydrolysis & 15 & 90 & $\begin{array}{l}239 \pm 81(\mathrm{~L}) 35 \\
\pm 7(\mathrm{D})\end{array}$ & $\begin{array}{l}\text { Reinforcing filler for } \\
\text { polymer composites }\end{array}$ & [135] \\
\hline Spruce bark & $\mathrm{H}_{2} \mathrm{SO}_{4}$ hydrolysis & 11 & 84 & $\begin{array}{ll}175.3 & (\mathrm{~L}) \\
2.8(\mathrm{D}) & \\
\end{array}$ & $\begin{array}{l}\text { Reinforcement agent } \\
\text { in nanocomposites. }\end{array}$ & {$[48]$} \\
\hline $\begin{array}{l}\text { Tetra pak fibers } \\
\text { waste }\end{array}$ & $\mathrm{H}_{2} \mathrm{SO}_{4}$ hydrolysis & $7-15$ & $64-83$ & $\begin{array}{l}207 \pm 78(\mathrm{~L}) \\
23 \pm 8(\mathrm{~W}) \\
\end{array}$ & 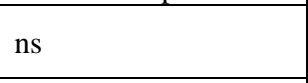 & $\begin{array}{l}{[136]} \\
{[137]}\end{array}$ \\
\hline Wood fireboard & $\begin{array}{lr}\text { Ethanol and } \\
\text { peroxide } \\
\text { ultrasonication }\end{array}$ & 24 & 71 & $\begin{array}{l}164.7(\mathrm{~L}) \\
6.7(\mathrm{D})\end{array}$ & $\begin{array}{l}\text { Reinforcement agent } \\
\text { for composites }\end{array}$ & [138] \\
\hline \multicolumn{7}{|c|}{ CNCs from technical cellulose } \\
\hline Sources & Extraction method & $\begin{array}{l}\text { Yield } \\
(\%)\end{array}$ & $\begin{array}{l}\mathrm{CrI} \\
(\%) \\
\end{array}$ & $\begin{array}{l}\text { Size }(n m) \\
W, L, D, T\end{array}$ & Applications & Ref. \\
\hline \multirow{3}{*}{$\begin{array}{l}\text { Bleached } \\
\text { Eucalyptus Kraft } \\
\text { pulp }\end{array}$} & $\begin{array}{l}\mathrm{H}_{2} \mathrm{SO}_{4} \\
\mathrm{CH}_{3} \mathrm{CO}_{2} \mathrm{H} \\
\text { hydrolysis }\end{array}$ & $64-79$ & $77-80$ & $\begin{array}{l}175-526(\mathrm{~L}) \\
9-23(\mathrm{~W})\end{array}$ & ns & [139] \\
\hline & $\begin{array}{l}\text { Enzymatic } \\
\text { hydrolysis and } \\
\text { mechanical } \\
\text { treatment at high } \\
\text { pressure }\end{array}$ & ns & 95 & $16.0(\mathrm{D})$ & $\begin{array}{l}\text { Aerogel absorber in } \\
\text { food packaging. }\end{array}$ & [71] \\
\hline & Organic acids & $2-25$ & $76-82$ & $\begin{array}{l}273-377(\mathrm{~L}) \\
15(\mathrm{D})\end{array}$ & $\begin{array}{l}\text { Biocomposite } \\
\text { applications }\end{array}$ & {$[55]$} \\
\hline \multirow[b]{2}{*}{$\begin{array}{l}\text { Microcrystalline } \\
\text { cellulose }\end{array}$} & $\begin{array}{lr}\text { Ionic } & \text { liquid } \\
\text { catalysis } & \text { and } \\
\text { solvolysis } & \\
\end{array}$ & ns & 95.5 & $\begin{array}{l}70-80(\mathrm{~L}) \\
15-20(\mathrm{D})\end{array}$ & $\begin{array}{lr}\text { Reinforcement } & \text { and } \\
\text { fillers } & \text { in } \\
\text { nanocomposites } & \\
\end{array}$ & [64] \\
\hline & $\begin{array}{l}\mathrm{H}_{2} \mathrm{SO}_{4} \text { hydrolysis } \\
\text { and hydrogen } \\
\text { peroxide } \\
\text { oxidation }\end{array}$ & ns & 77.3 & $460.5(\mathrm{D})$ & $\begin{array}{l}\text { Emulsifiers for } \\
\text { medical and cosmetic } \\
\text { uses }\end{array}$ & [140] \\
\hline $\begin{array}{l}\text { Softwood } \\
\text { sulphite pulp }\end{array}$ & $\begin{array}{l}\text { Ultrasonic } \\
\text { assisted } \mathrm{H}_{2} \mathrm{SO}_{4} \\
\text { hydrolysis }\end{array}$ & 71 & 81 & $\begin{array}{l}41.4(\mathrm{~L}) \\
5.9(\mathrm{D}) \\
4.1(\mathrm{~T})\end{array}$ & $\begin{array}{l}\text { Nanoreinforcing } \\
\text { agents in polymers }\end{array}$ & {$[14]$} \\
\hline
\end{tabular}

${ }^{1} \mathrm{I}_{\mathrm{C}}$ : crystallinity index; ${ }^{2} \mathrm{~L}$ : length; ${ }^{3} \mathrm{~W}$ : width; ${ }^{4} \mathrm{D}$ :diameter; ${ }^{5} \mathrm{PS}$ : particle size; ${ }^{6} \mathrm{~T}$ : thickness. 


\section{Conclusions}

Numerous non-edible plant biomass and agro-industrial wastes in any part of the world represent viable and sustainable cellulose sources from which CNCs can be obtained. As demonstrated in this work, very diverse processes have been explored to define methodologies more effective, less expensive, environmentally friendly, and precise for each source studied. Recent research in CNCs has unraveled excellent properties, which have made them an essential resource for the manufacture of advanced materials. Properties such as large surface area, reactive and tunable functional-groups, mechanical strength, and thermal stability make $\mathrm{CNCs}$ an attractive option for manufacturing biocompatible, biodegradable, and renewable nanostructured materials.

Another reason to continue focusing efforts to establish better processes for obtaining CNCs, is its impact on society. The sustainable exploitation of renewable resources and agroindustrial wastes to achieve an added value product contributes to scientific and technological progress. Indeed, it could promote an economic and social benefit for many communities all around the world.

\section{Funding}

This research was funded by Universidad Autónoma de Coahuila through the Program “Proyectos Semilla 2020", grant 046. T.O., thanks for the scholarship received.

\section{Acknowledgments}

This research has no acknowledgment.

\section{Conflicts of Interest}

The authors declare no conflict of interest.

\section{References}

1. Thomas, B.; Raj, M.C.; B, A.K.; H, R.M.; Joy, J.; Moores, A.; Drisko, G.L.; Sanchez, C. Nanocellulose, a Versatile Green Platform: From Biosources to Materials and Their Applications. Chemical Reviews 2018, 118, 11575-11625, https://doi.org/10.1021/acs.chemrev.7b00627.

2. Dufresne, A. Nanocellulose: From Nature to High Performance Tailored Materials. Berlin, Boston: DE GRUYTER. 2012; https://doi.org/10.1515/9783110254600.

3. Thomas, P.; Duolikun, T.; Rumjit, N.P.; Moosavi, S.; Lai, C.W.; Bin Johan, M.R.; Fen, L.B. Comprehensive review on nanocellulose: Recent developments, challenges and future prospects. Journal of the Mechanical Behavior of Biomedical Materials 2020, 110, https://doi.org/10.1016/j.jmbbm.2020.103884.

4. Rajinipriya, M.; Nagalakshmaiah, M.; Robert, M.; Elkoun, S. Importance of Agricultural and Industrial Waste in the Field of Nanocellulose and Recent Industrial Developments of Wood Based Nanocellulose: A Review. ACS Sustainable Chemistry \& Engineering 2018, 6, 2807-2828, https://doi.org/10.1021/acssuschemeng.7b03437.

5. Ng, H.-M.; Sin, L.T.; Tee, T.-T.; Bee, S.-T.; Hui, D.; Low, C.-Y.; Rahmat, A.R. Extraction of cellulose nanocrystals from plant sources for application as reinforcing agent in polymers. Composites Part B: Engineering 2015, 75, 176-200, https://doi.org/10.1016/j.compositesb.2015.01.008.

6. García, A.; Gandini, A.; Labidi, J.; Belgacem, N.; Bras, J. Industrial and crop wastes: A new source for nanocellulose biorefinery. Industrial Crops and Products 2016, 93, 26-38, https://doi.org/10.1016/j.indcrop.2016.06.004.

7. Trache, D.; Tarchoun, A.F.; Derradji, M.; Hamidon, T.S.; Masruchin, N.; Brosse, N.; Hussin, M.H. Nanocellulose: From Fundamentals to Advanced Applications. 2020, 8, https://doi.org/10.3389/fchem.2020.00392.

8. Foster, E.J.; Moon, R.J.; Agarwal, U.P.; Bortner, M.J.; Bras, J.; Camarero-Espinosa, S.; Chan, K.J.; Clift, M.J.D.; Cranston, E.D.; Eichhorn, S.J.; Fox, D.M.; Hamad, W.Y.; Heux, L.; Jean, B.; Korey, M.; Nieh, W.; 
Ong, K.J.; Reid, M.S.; Renneckar, S.; Roberts, R.; Shatkin, J.A.; Simonsen, J.; Stinson-Bagby, K.; Wanasekara, N.; Youngblood, J. Current characterization methods for cellulose nanomaterials. Chemical Society Reviews 2018, 47, 2609-2679, https://doi.org/10.1039/C6CS00895J.

9. Klemm, D.; Kramer, F.; Moritz, S.; Lindström, T.; Ankerfors, M.; Gray, D.; Dorris, A. Nanocelluloses: A New Family of Nature-Based Materials. Angewandte Chemie International Edition 2011, 50, 5438-5466, https://doi.org/10.1002/anie.201001273.

10. Klemm, D.; Cranston, E.D.; Fischer, D.; Gama, M.; Kedzior, S.A.; Kralisch, D.; Kramer, F.; Kondo, T.; Lindström, T.; Nietzsche, S.; Petzold-Welcke, K.; Rauchfuß, F. Nanocellulose as a natural source for groundbreaking applications in materials science: Today's state. Materials Today 2018, 21, 720-748, https://doi.org/10.1016/j.mattod.2018.02.001.

11. Eichhorn, S.J.; Dufresne, A.; Aranguren, M.; Marcovich, N.E.; Capadona, J.R.; Rowan, S.J.; Weder, C.; Thielemans, W.; Roman, M.; Renneckar, S.; Gindl, W.; Veigel, S.; Keckes, J.; Yano, H.; Abe, K.; Nogi, M.; Nakagaito, A.N.; Mangalam, A.; Simonsen, J.; Benight, A.S.; Bismarck, A.; Berglund, L.A.; Peijs, T. Review: current international research into cellulose nanofibres and nanocomposites. Journal of Materials Science 2010, 45, 1-33, https://doi.org/10.1007/s10853-009-3874-0.

12. Kamel, R.; El-Wakil, N.A.; Dufresne, A.; Elkasabgy, N.A. Nanocellulose: From an agricultural waste to a valuable pharmaceutical ingredient. International Journal of Biological Macromolecules 2020, 163, 15791590, https://doi.org/10.1016/j.ijbiomac.2020.07.242.

13. Thakur, M.; Sharma, A.; Ahlawat, V.; Bhattacharya, M.; Goswami, S. Process optimization for the production of cellulose nanocrystals from rice straw derived $\alpha$-cellulose. Materials Science for Energy Technologies 2020, 3, 328-334, https://doi.org/10.1016/j.mset.2019.12.005.

14. Guo, J.; Guo, X.; Wang, S.; Yin, Y. Effects of ultrasonic treatment during acid hydrolysis on the yield, particle size and structure of cellulose nanocrystals. Carbohydrate Polymers 2016, 135, 248-255, https://doi.org/10.1016/j.carbpol.2015.08.068.

15. Siqueira, G.; Tapin-Lingua, S.; Bras, J.; da Silva Perez, D.; Dufresne, A. Mechanical properties of natural rubber nanocomposites reinforced with cellulosic nanoparticles obtained from combined mechanical shearing, and enzymatic and acid hydrolysis of sisal fibers. Cellulose 2011, 18, 57-65, https://doi.org/10.1007/s10570-010-9463-1.

16. Lu, Q.; Lin, W.; Tang, L.; Wang, S.; Chen, X.; Huang, B. A mechanochemical approach to manufacturing bamboo cellulose nanocrystals. Journal of Materials Science 2015, 50, 611-619, https://doi.org/10.1007/s10853-014-8620-6.

17. Douard, L.; Bras, J.; Encinas, T.; Belgacem, M.N. Natural acidic deep eutectic solvent to obtain cellulose nanocrystals using the design of experience approach. Carbohydrate Polymers 2021, 252, https://doi.org/10.1016/j.carbpol.2020.117136.

18. Novo, L.P.; Bras, J.; García, A.; Belgacem, N.; Curvelo, A.A.S. Subcritical Water: A Method for Green Production of Cellulose Nanocrystals. ACS Sustainable Chemistry \& Engineering 2015, 3, 2839-2846, https://doi.org/10.1021/acssuschemeng.5b00762.

19. Nechyporchuk, O.; Belgacem, M.N.; Bras, J. Production of cellulose nanofibrils: A review of recent advances. Industrial Crops and Products 2016, 93, 2-25, https://doi.org/10.1016/j.indcrop.2016.02.016.

20. Moon, R.J.; Martini, A.; Nairn, J.; Simonsen, J.; Youngblood, J. Cellulose nanomaterials review: structure, properties and nanocomposites. Chemical Society Reviews 2011, 40, 3941-3994, https://doi.org/10.1039/c0cs00108b.

21. Gatenholm, P.; Klemm, D. Bacterial Nanocellulose as a Renewable Material for Biomedical Applications. MRS Bulletin 2010, 35, 208-213, https://doi.org/10.1557/mrs2010.653.

22. Tappi, T.A. for the P. and P. I. (2011, October 24). Roadmap for the Development of International Standards for Nanocellulose. October 24, 2011.

23. Jackson, J.K.; Letchford, K.; Wasserman, B.Z.; Ye, L.; Hamad, W.Y.; Burt, H.M. The use of nanocrystalline cellulose for the binding and controlled release of drugs. Int J Nanomedicine 2011, 6, 321-330, https://doi.org/10.2147/IJN.S16749.

24. Ghorbani, M.; Roshangar, L.; Soleimani Rad, J. Development of reinforced chitosan/pectin scaffold by using the cellulose nanocrystals as nanofillers: An injectable hydrogel for tissue engineering. European Polymer Journal 2020, 130, https://doi.org/10.1016/j.eurpolymj.2020.109697.

25. Ganguly, K.; Patel, D.K.; Dutta, S.D.; Shin, W.-C.; Lim, K.-T. Stimuli-responsive self-assembly of cellulose nanocrystals (CNCs): Structures, functions, and biomedical applications. International Journal of Biological Macromolecules 2020, 155, 456-469, https://doi.org/10.1016/j.ijbiomac.2020.03.171.

26. Gómez H, C.; Serpa, A.; Velásquez-Cock, J.; Gañán, P.; Castro, C.; Vélez, L.; Zuluaga, R. Vegetable nanocellulose in food science: A review. Food Hydrocolloids 2016, 57, 178-186, https://doi.org/10.1016/j.foodhyd.2016.01.023.

27. He, Y.; Li, H.; Fei, X.; Peng, L. Carboxymethyl cellulose/cellulose nanocrystals immobilized silver nanoparticles as an effective coating to improve barrier and antibacterial properties of paper for food packaging applications. Carbohydrate Polymers 2021, 252, https://doi.org/10.1016/j.carbpol.2020.117156.

28. Azeredo, H.M.C.; Rosa, M.F.; Mattoso, L.H.C. Nanocellulose in bio-based food packaging applications. Industrial Crops and Products 2017, 97, 664-671, https://doi.org/10.1016/j.indcrop.2016.03.013. 
29. Leite, L.S.F.; Bilatto, S.; Paschoalin, R.T.; Soares, A.C.; Moreira, F.K.V.; Oliveira, O.N.; Mattoso, L.H.C.; Bras, J. Eco-friendly gelatin films with rosin-grafted cellulose nanocrystals for antimicrobial packaging. International Journal of Biological Macromolecules 2020, 165, 2974-2983, https://doi.org/10.1016/j.ijbiomac.2020.10.189.

30. Leite, L.S.F.; Moreira, F.K.V.; Mattoso, L.H.C.; Bras, J. Electrostatic interactions regulate the physical properties of gelatin-cellulose nanocrystals nanocomposite films intended for biodegradable packaging. Food Hydrocolloids 2020, https://doi.org/10.1016/j.foodhyd.2020.106424.

31. Belbekhouche, S.; Bras, J.; Siqueira, G.; Chappey, C.; Lebrun, L.; Khelifi, B.; Marais, S.; Dufresne, A. Water sorption behavior and gas barrier properties of cellulose whiskers and microfibrils films. Carbohydrate Polymers 2011, 83, 1740-1748, https://doi.org/10.1016/j.carbpol.2010.10.036.

32. Alonso-Lerma, B.; Barandiaran, L.; Ugarte, L.; Larraza, I.; Reifs, A.; Olmos-Juste, R.; Barruetabeña, N.; Amenabar, I.; Hillenbrand, R.; Eceiza, A.; Perez-Jimenez, R. High performance crystalline nanocellulose using an ancestral endoglucanase. Communications Materials 2020, 1, https://doi.org/10.1038/s43246-02000055-5.

33. Li, S.; Lee, P.S. Development and applications of transparent conductive nanocellulose paper. Science and Technology of Advanced Materials 2017, 18, 620-633, https://doi.org/10.1080/14686996.2017.1364976.

34. Tran, A.; Boott, C.E.; MacLachlan, M.J. Understanding the Self-Assembly of Cellulose NanocrystalsToward Chiral Photonic Materials. Advanced Materials 2020, 32, https://doi.org/10.1002/adma.201905876.

35. Nakagaito, A.N.; Nogi, M.; Yano, H. Displays from Transparent Films of Natural Nanofibers. MRS Bulletin 2010, 35, 214-218, https://doi.org/10.1557/mrs2010.654.

36. Peng, B.L.; Dhar, N.; Liu, H.L.; Tam, K.C. Chemistry and applications of nanocrystalline cellulose and its derivatives: A nanotechnology perspective. The Canadian Journal of Chemical Engineering 2011, 89, 11911206, https://doi.org/10.1002/cjce.20554.

37. Dufresne, A. Cellulose nanomaterial reinforced polymer nanocomposites. Current Opinion in Colloid \& Interface Science 2017, 29, 1-8, https://doi.org/10.1016/j.cocis.2017.01.004.

38. Kargarzadeh, H.; Mariano, M.; Huang, J.; Lin, N.; Ahmad, I.; Dufresne, A.; Thomas, S. Recent developments on nanocellulose reinforced polymer nanocomposites: A review. Polymer 2017, 132, 368393, https://doi.org/10.1016/j.polymer.2017.09.043.

39. Mishra, R.K.; Sabu, A.; Tiwari, S.K. Materials chemistry and the futurist eco-friendly applications of nanocellulose: Status and prospect. Journal of Saudi Chemical Society 2018, 22, 949-978, https://doi.org/10.1016/j.jscs.2018.02.005.

40. Börjesson, M.; Westman, G. Crystalline Nanocellulose - Preparation, Modification, and Properties. In: Cellulose - Fundamental Aspects and Current Trends. Poletto, M.; Ornaghi, H.L. (Eds.), InTech. 2015; https://doi.org/10.5772/61899.

41. Lu, P.; Hsieh, Y.-L. Preparation and characterization of cellulose nanocrystals from rice straw. Carbohydrate Polymers 2012, 87, 564-573, https://doi.org/10.1016/j.carbpol.2011.08.022.

42. Flauzino Neto, W.P.; Silvério, H.A.; Dantas, N.O.; Pasquini, D. Extraction and characterization of cellulose nanocrystals from agro-industrial residue - Soy hulls. Industrial Crops and Products 2013, 42, 480-488, https://doi.org/10.1016/j.indcrop.2012.06.041.

43. Jiang, Q.; Xing, X.; Jing, Y.; Han, Y. Preparation of cellulose nanocrystals based on waste paper via different systems. International Journal of Biological Macromolecules 2020, 149, 1318-1322, https://doi.org/10.1016/j.ijbiomac.2020.02.110.

44. Nascimento, S.A.; Rezende, C.A. Combined approaches to obtain cellulose nanocrystals, nanofibrils and fermentable sugars from elephant grass. Carbohydrate Polymers 2018, 180, 38-45, https://doi.org/10.1016/j.carbpol.2017.09.099.

45. Liu, Z.; Li, X.; Xie, W.; Deng, H. Extraction, isolation and characterization of nanocrystalline cellulose from industrial kelp (Laminaria japonica) waste. Carbohydrate Polymers 2017, 173, 353-359, https://doi.org/10.1016/j.carbpol.2017.05.079.

46. Ilyas, R.A.; Sapuan, S.M.; Ishak, M.R. Isolation and characterization of nanocrystalline cellulose from sugar palm fibres (Arenga Pinnata). Carbohydrate Polymers 2018, 181, 1038-1051, https://doi.org/10.1016/j.carbpol.2017.11.045.

47. Yu, H.; Qin, Z.; Liang, B.; Liu, N.; Zhou, Z.; Chen, L. Facile extraction of thermally stable cellulose nanocrystals with a high yield of $93 \%$ through hydrochloric acid hydrolysis under hydrothermal conditions. Journal of Materials Chemistry A 2013, 1, 3938-3944, https://doi.org/10.1039/c3ta01150j.

48. Le Normand, M.; Moriana, R.; Ek, M. Isolation and characterization of cellulose nanocrystals from spruce bark in a biorefinery perspective. Carbohydrate Polymers 2014, 111, 979-987, https://doi.org/10.1016/j.carbpol.2014.04.092.

49. Kassab, Z.; Syafri, E.; Tamraoui, Y.; Hannache, H.; Qaiss, A.E.K.; El Achaby, M. Characteristics of sulfated and carboxylated cellulose nanocrystals extracted from Juncus plant stems. International Journal of Biological Macromolecules 2020, 154, 1419-1425, https://doi.org/10.1016/j.ijbiomac.2019.11.023.

50. Kassab, Z.; Abdellaoui, Y.; Salim, M.H.; Bouhfid, R.; Qaiss, A.E.K.; El Achaby, M. Micro- and nanocelluloses derived from hemp stalks and their effect as polymer reinforcing materials. Carbohydrate Polymers 2020, 245, https://doi.org/10.1016/j.carbpol.2020.116506. 
51. Camarero Espinosa, S.; Kuhnt, T.; Foster, E.J.; Weder, C. Isolation of Thermally Stable Cellulose Nanocrystals by Phosphoric Acid Hydrolysis. Biomacromolecules 2013, 14, 1223-1230, https://doi.org/10.1021/bm400219u.

52. Doh, H.; Lee, M.H.; Whiteside, W.S. Physicochemical characteristics of cellulose nanocrystals isolated from seaweed biomass. Food Hydrocolloids 2020, 102, https://doi.org/10.1016/j.foodhyd.2019.105542.

53. Kandhola, G.; Djioleu, A.; Rajan, K.; Labbé, N.; Sakon, J.; Carrier, D.J.; Kim, J.-W. Maximizing production of cellulose nanocrystals and nanofibers from pre-extracted loblolly pine kraft pulp: a response surface approach. Bioresources and Bioprocessing 2020, 7, https://doi.org/10.1186/s40643-020-00302-0.

54. Robles, E.; Izaguirre, N.; Dogaru, B.-I.; Popescu, C.-M.; Barandiaran, I.; Labidi, J. Sonochemical production of nanoscaled crystalline cellulose using organic acids. Green Chemistry 2020, 22, 4627-4639, https://doi.org/10.1039/D0GC01283A.

55. Chen, L.; Zhu, J.Y.; Baez, C.; Kitin, P.; Elder, T. Highly thermal-stable and functional cellulose nanocrystals and nanofibrils produced using fully recyclable organic acids. Green Chemistry 2016, 18, 3835-3843, https://doi.org/10.1039/C6GC00687F.

56. Li, D.; Henschen, J.; Ek, M. Esterification and hydrolysis of cellulose using oxalic acid dihydrate in a solvent-free reaction suitable for preparation of surface-functionalised cellulose nanocrystals with high yield. Green Chemistry 2017, 19, 5564-5567, https://doi.org/10.1039/C7GC02489D.

57. Yu, H.-Y.; Zhang, D.-Z.; Lu, F.-F.; Yao, J. New Approach for Single-Step Extraction of Carboxylated Cellulose Nanocrystals for Their Use As Adsorbents and Flocculants. ACS Sustainable Chemistry \& Engineering 2016, 4, 2632-2643, https://doi.org/10.1021/acssuschemeng.6b00126.

58. Du, H.; Liu, W.; Zhang, M.; Si, C.; Zhang, X.; Li, B. Cellulose nanocrystals and cellulose nanofibrils based hydrogels for biomedical applications. Carbohydrate Polymers 2019, 209, 130-144, https://doi.org/10.1016/j.carbpol.2019.01.020.

59. Lu, Q.; Cai, Z.; Lin, F.; Tang, L.; Wang, S.; Huang, B. Extraction of Cellulose Nanocrystals with a High Yield of $88 \%$ by Simultaneous Mechanochemical Activation and Phosphotungstic Acid Hydrolysis. ACS

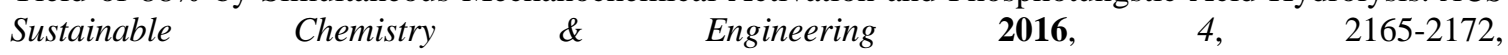
https://doi.org/10.1021/acssuschemeng.5b01620.

60. Lu, Q.; Cai, Z.; Lin, F.; Tang, L.; Wang, S.; Huang, B. Extraction of Cellulose Nanocrystals with a High Yield of $88 \%$ by Simultaneous Mechanochemical Activation and Phosphotungstic Acid Hydrolysis. ACS Sustainable Chemistry \& Engineering 2016, 4, 2165-2172, https://doi.org/10.1016/j.carbpol.2019.115114.

61. Cao, X.; Ding, B.; Yu, J.; Al-Deyab, S.S. Cellulose nanowhiskers extracted from TEMPO-oxidized jute fibers. Carbohydrate Polymers 2012, 90, 1075-1080, https://doi.org/10.1016/j.carbpol.2012.06.046.

62. Zhou, Y.; Saito, T.; Bergström, L.; Isogai, A. Acid-Free Preparation of Cellulose Nanocrystals by TEMPO Oxidation and Subsequent Cavitation. Biomacromolecules 2018, 19, 633-639, https://doi.org/10.1021/acs.biomac.7b01730,

63. Abushammala, H.; Krossing, I.; Laborie, M.-P. Ionic liquid-mediated technology to produce cellulose nanocrystals directly from wood. Carbohydrate Polymers 2015, 134, 609-616, https://doi.org/10.1016/j.carbpol.2015.07.079.

64. Tan, X.Y.; Abd Hamid, S.B.; Lai, C.W. Preparation of high crystallinity cellulose nanocrystals (CNCs) by ionic liquid solvolysis. Biomass and Bioenergy 2015, 81, 584-591, https://doi.org/10.1016/j.biombioe.2015.08.016.

65. Elqady, H.I.; El-Shazly, A.H.; El-Kady, M.F.; Madih, K. Assessment of New Technique for Production Cellulose Nanocrystals from Agricultural Waste. Materials Science Forum 2018, 928, 83-88, https://doi.org/10.4028/www.scientific.net/MSF.928.83.

66. Hemmati, F.; Jafari, S.M.; Kashaninejad, M.; Barani Motlagh, M. Synthesis and characterization of cellulose nanocrystals derived from walnut shell agricultural residues. International Journal of Biological Macromolecules 2018, 120, 1216-1224, https://doi.org/10.1016/j.ijbiomac.2018.09.012.

67. Melikoğlu, A.Y.; Bilek, S.E.; Cesur, S. Optimum alkaline treatment parameters for the extraction of cellulose and production of cellulose nanocrystals from apple pomace. Carbohydrate Polymers 2019, 215, 330-337, https://doi.org/10.1016/j.carbpol.2019.03.103.

68. Kamelnia, E.; Divsalar, A.; Darroudi, M.; Yaghmaei, P.; Sadri, K. Production of new cellulose nanocrystals from Ferula gummosa and their use in medical applications via investigation of their biodistribution. Industrial Crops and Products 2019, 139, https://doi.org/10.1016/j.indcrop.2019.111538.

69. Gao, A.; Chen, H.; Tang, J.; Xie, K.; Hou, A. Efficient extraction of cellulose nanocrystals from waste Calotropis gigantea fiber by SO42-/TiO2 nano-solid superacid catalyst combined with ball milling exfoliation. Industrial Crops and Products 2020, 152, https://doi.org/10.1016/j.indcrop.2020.112524.

70. Anderson, S.R.; Esposito, D.; Gillette, W.; Zhu, J.Y.; Baxa, U.; Mcneil, S.E. Enzymatic preparation of nanocrystalline and microcrystalline cellulose. TAPPI Journal 2014, 13, 35-42, https://doi.org/10.32964/TJ13.5.35.

71. de Oliveira, J.P.; Bruni, G.P.; el Halal, S.L.M.; Bertoldi, F.C.; Dias, A.R.G.; Zavareze, E.d.R. Cellulose nanocrystals from rice and oat husks and their application in aerogels for food packaging. International Journal of Biological Macromolecules 2019, 124, 175-184, https://doi.org/10.1016/j.ijbiomac.2018.11.205. 
72. Pereira, B.; Arantes, V. Production of cellulose nanocrystals integrated into a biochemical sugar platform process via enzymatic hydrolysis at high solid loading. Industrial Crops and Products 2020, 152, https://doi.org/10.1016/j.indcrop.2020.112377.

73. Espino, E.; Cakir, M.; Domenek, S.; Román-Gutiérrez, A.D.; Belgacem, N.; Bras, J. Isolation and characterization of cellulose nanocrystals from industrial by-products of Agave tequilana and barley. Industrial Crops and Products 2014, 62, 552-559, https://doi.org/10.1016/j.indcrop.2014.09.017.

74. Pech-Cohuo, S.-C.; Canche-Escamilla, G.; Valadez-González, A.; Fernández-Escamilla, V.V.A.; UribeCalderon, J. Production and Modification of Cellulose Nanocrystals from Agave tequilana Weber Waste and Its Effect on the Melt Rheology of PLA. International Journal of Polymer Science 2018, 2018, 1-14, https://doi.org/10.1155/2018/3567901.

75. Wang, W.; Du, G.; Li, C.; Zhang, H.; Long, Y.; Ni, Y. Preparation of cellulose nanocrystals from asparagus (Asparagus officinalis L.) and their applications to palm oil/water Pickering emulsion. Carbohydrate Polymers 2016, 151, 1-8, https://doi.org/10.1016/j.carbpol.2016.05.052.

76. Brito, B.S.L.; Pereira, F.V.; Putaux, J.-L.; Jean, B. Preparation, morphology and structure of cellulose nanocrystals from bamboo fibers. Cellulose 2012, 19, 1527-1536, https://doi.org/10.1007/s10570-012-97389 .

77. Wijaya, C.J.; Ismadji, S.; Aparamarta, H.W.; Gunawan, S. Optimization of cellulose nanocrystals from bamboo shoots using Response Surface Methodology. Heliyon 2019, 5, https://doi.org/10.1016/j.heliyon.2019.e02807.

78. Pereira, A.L.S.; Nascimento, D.M.d.; Souza Filho, M.d.s.M.; Morais, J.P.S.; Vasconcelos, N.F.; Feitosa, J.P.A.; Brígida, A.I.S.; Rosa, M.d.F. Improvement of polyvinyl alcohol properties by adding nanocrystalline cellulose isolated from banana pseudostems. Carbohydrate Polymers 2014, 112, 165-172, https://doi.org/10.1016/j.carbpol.2014.05.090.

79. Oun, A.A.; Rhim, J.-W. Isolation of cellulose nanocrystals from grain straws and their use for the preparation of carboxymethyl cellulose-based nanocomposite films. Carbohydrate Polymers 2016, 150, 187-200, https://doi.org/10.1016/j.carbpol.2016.05.020.

80. Fortunati, E.; Benincasa, P.; Balestra, G.M.; Luzi, F.; Mazzaglia, A.; Del Buono, D.; Puglia, D.; Torre, L. Revalorization of barley straw and husk as precursors for cellulose nanocrystals extraction and their effect on PVA_CH nanocomposites. Industrial Crops and Products 2016, 92, 201-217, https://doi.org/10.1016/j.indcrop.2016.07.047.

81. Sá, N.M.S.M.; Mattos, A.L.A.; Silva, L.M.A.; Brito, E.S.; Rosa, M.F.; Azeredo, H.M.C. From cashew byproducts to biodegradable active materials: Bacterial cellulose-lignin-cellulose nanocrystal nanocomposite films. International Journal of Biological Macromolecules 2020, 161, 1337-1345, https://doi.org/10.1016/j.ijbiomac.2020.07.269.

82. Collazo-Bigliardi, S.; Ortega-Toro, R.; Chiralt Boix, A. Isolation and characterisation of microcrystalline cellulose and cellulose nanocrystals from coffee husk and comparative study with rice husk. Carbohydrate Polymers 2018, 191, 205-215, https://doi.org/10.1016/j.carbpol.2018.03.022.

83. Silvério, H.A.; Flauzino Neto, W.P.; Pasquini, D. Effect of Incorporating Cellulose Nanocrystals from Corncob on the Tensile, Thermal and Barrier Properties of Poly(Vinyl Alcohol) Nanocomposites. Journal of Nanomaterials 2013, 2013, 1-9, https://doi.org/10.1155/2013/289641.

84. Louis, A.C.F.; Venkatachalam, S. Energy efficient process for valorization of corn cob as a source for nanocrystalline cellulose and hemicellulose production. International Journal of Biological Macromolecules 2020, 163, 260-269, https://doi.org/10.1016/j.ijbiomac.2020.06.276.

85. Rahbar Shamskar, K.; Heidari, H.; Rashidi, A. Preparation and evaluation of nanocrystalline cellulose aerogels from raw cotton and cotton stalk. Industrial Crops and Products 2016, 93, 203-211, https://doi.org/10.1016/j.indcrop.2016.01.044.

86. Morais, J.P.S.; Rosa, M.d.F.; de Souza Filho, M.d.s.M.; Nascimento, L.D.; do Nascimento, D.M.; Cassales, A.R. Extraction and characterization of nanocellulose structures from raw cotton linter. Carbohydrate Polymers 2013, 91, 229-235, https://doi.org/10.1016/j.carbpol.2012.08.010.

87. Sai Prasanna, N.; Mitra, J. Isolation and characterization of cellulose nanocrystals from Cucumis sativus peels. Carbohydrate Polymers 2020, 247, https://doi.org/10.1016/j.carbpol.2020.116706.

88. Alothman, O.Y.; Kian, L.K.; Saba, N.; Jawaid, M.; Khiari, R. Cellulose nanocrystal extracted from date palm fibre: Morphological, structural and thermal properties. Industrial Crops and Products 2021, 159, https://doi.org/10.1016/j.indcrop.2020.113075.

89. Prasad Reddy, J.; Rhim, J.-W. Isolation and characterization of cellulose nanocrystals from garlic skin. Materials Letters 2014, 129, 20-23, https://doi.org/10.1016/j.matlet.2014.05.019.

90. Kallel, F.; Bettaieb, F.; Khiari, R.; García, A.; Bras, J.; Chaabouni, S.E. Isolation and structural characterization of cellulose nanocrystals extracted from garlic straw residues. Industrial Crops and Products 2016, 87, 287-296, https://doi.org/10.1016/j.indcrop.2016.04.060.

91. Coelho, C.C.d.S.; Silva, R.B.S.; Carvalho, C.W.P.; Rossi, A.L.; Teixeira, J.A.; Freitas-Silva, O.; Cabral, L.M.C. Cellulose nanocrystals from grape pomace and their use for the development of starch-based nanocomposite films. International Journal of Biological Macromolecules 2020, 159, 1048-1061, https://doi.org/10.1016/j.ijbiomac.2020.05.046. 
92. Sucaldito, M.R.; Camacho, D.H. Characteristics of unique HBr-hydrolyzed cellulose nanocrystals from freshwater green algae (Cladophora rupestris) and its reinforcement in starch-based film. Carbohydrate Polymers 2017, 169, 315-323, https://doi.org/10.1016/j.carbpol.2017.04.031.

93. Fortunati, E.; Puglia, D.; Luzi, F.; Santulli, C.; Kenny, J.M.; Torre, L. Binary PVA bio-nanocomposites containing cellulose nanocrystals extracted from different natural sources: Part I. Carbohydrate Polymers 2013, 97, 825-836, https://doi.org/10.1016/j.carbpol.2013.03.075.

94. Luzi, F.; Fortunati, E.; Jiménez, A.; Puglia, D.; Pezzolla, D.; Gigliotti, G.; Kenny, J.M.; Chiralt, A.; Torre, L. Production and characterization of PLA_PBS biodegradable blends reinforced with cellulose nanocrystals extracted from hemp fibres. Industrial Crops and Products 2016, 93, 276-289, https://doi.org/10.1016/j.indcrop.2016.01.045.

95. Jiang, Y.; Zhou, J.; Zhang, Q.; Zhao, G.; Heng, L.; Chen, D.; Liu, D. Preparation of cellulose nanocrystals from Humulus japonicus stem and the influence of high temperature pretreatment. Carbohydrate Polymers 2017, 164, 284-293, https://doi.org/10.1016/j.carbpol.2017.02.021.

96. Mohamed, M.A.; W. Salleh, W.N.; Jaafar, J.; Ismail, A.F.; Abd Mutalib, M.; Mohamad, A.B.; M. Zain, M.F.; Awang, N.A.; Mohd Hir, Z.A. Physicochemical characterization of cellulose nanocrystal and nanoporous self-assembled CNC membrane derived from Ceiba pentandra. Carbohydrate Polymers 2017, 157, 1892-1902, https://doi.org/10.1016/j.carbpol.2016.11.078.

97. Lu, Q.-1.; Tang, L.-r.; Wang, S.; Huang, B.; Chen, Y.-d.; Chen, X.-r. An investigation on the characteristics of cellulose nanocrystals from Pennisetum sinese. Biomass and Bioenergy 2014, 70, 267-272, https://doi.org/10.1016/j.biombioe.2014.09.012.

98. Wang, Z.; Yao, Z.; Zhou, J.; He, M.; Jiang, Q.; Li, S.; Ma, Y.; Liu, M.; Luo, S. Isolation and characterization of cellulose nanocrystals from pueraria root residue. International Journal of Biological Macromolecules 2019, 129, 1081-1089, https://doi.org/10.1016/j.ijbiomac.2018.07.055.

99. Sheltami, R.M.; Abdullah, I.; Ahmad, I.; Dufresne, A.; Kargarzadeh, H. Extraction of cellulose nanocrystals from mengkuang leaves (Pandanus tectorius). Carbohydrate Polymers 2012, 88, 772-779, https://doi.org/10.1016/j.carbpol.2012.01.062.

100. Cudjoe, E.; Hunsen, M.; Xue, Z.; Way, A.E.; Barrios, E.; Olson, R.A.; Hore, M.J.A.; Rowan, S.J. Miscanthus Giganteus: A commercially viable sustainable source of cellulose nanocrystals. Carbohydrate Polymers 2017, 155, 230-241, https://doi.org/10.1016/j.carbpol.2016.08.049.

101. Lamaming, J.; Hashim, R.; Sulaiman, O.; Leh, C.P.; Sugimoto, T.; Nordin, N.A. Cellulose nanocrystals isolated from oil palm trunk. Carbohydrate Polymers 2015, 127, 202-208, https://doi.org/10.1016/j.carbpol.2015.03.043.

102. Fortunati, E.; Puglia, D.; Monti, M.; Santulli, C.; Maniruzzaman, M.; Kenny, J.M. Cellulose nanocrystals extracted from okra fibers in PVA nanocomposites. Journal of Applied Polymer Science 2013, 128, 32203230.

103. Rhim, J.-W.; Reddy, J.P.; Luo, X. Isolation of cellulose nanocrystals from onion skin and their utilization for the preparation of agar-based bio-nanocomposites films. Cellulose 2015, 22, 407-420, https://doi.org/10.1007/s10570-014-0517-7.

104. Wijaya, C.J.; Saputra, S.N.; Soetaredjo, F.E.; Putro, J.N.; Lin, C.X.; Kurniawan, A.; Ju, Y.-H.; Ismadji, S. Cellulose nanocrystals from passion fruit peels waste as antibiotic drug carrier. Carbohydrate Polymers 2017, 175, 370-376, https://doi.org/10.1016/j.carbpol.2017.08.004.

105. Santos, R.M.d.; Flauzino Neto, W.P.; Silvério, H.A.; Martins, D.F.; Dantas, N.O.; Pasquini, D. Cellulose nanocrystals from pineapple leaf, a new approach for the reuse of this agro-waste. Industrial Crops and Products 2013, 50, 707-714, https://doi.org/10.1016/j.indcrop.2013.08.049.

106. Kasiri, N.; Fathi, M. Production of cellulose nanocrystals from pistachio shells and their application for stabilizing Pickering emulsions. International Journal of Biological Macromolecules 2018, 106, 1023-1031, https://doi.org/10.1016/j.ijbiomac.2017.08.112.

107. Li, H.; Shi, H.; He, Y.; Fei, X.; Peng, L. Preparation and characterization of carboxymethyl cellulose-based composite films reinforced by cellulose nanocrystals derived from pea hull waste for food packaging applications. International Journal of Biological Macromolecules 2020, 164, 4104-4112, https://doi.org/10.1016/j.ijbiomac.2020.09.010.

108. Bano, S., \& Negi, Y. S. (2017). Studies on cellulose nanocrystals isolated from groundnut shells. Carbohydrate Polymers, 157, 1041-1049. https://doi.org/10.1016/j.carbpol.2016.10.069.

109. Olad, A.; Doustdar, F.; Gharekhani, H. Fabrication and characterization of a starch-based superabsorbent hydrogel composite reinforced with cellulose nanocrystals from potato peel waste. Colloids and Surfaces A: Physicochemical and Engineering Aspects 2020, 601, https://doi.org/10.1016/j.colsurfa.2020.124962.

110. Kusmono; Listyanda, R.F.; Wildan, M.W.; Ilman, M.N. Preparation and characterization of cellulose nanocrystal extracted from ramie fibers by sulfuric acid hydrolysis. Heliyon 2020, 6, https://doi.org/10.1016/j.heliyon.2020.e05486.

111. El Achaby, M.; Kassab, Z.; Aboulkas, A.; Gaillard, C.; Barakat, A. Reuse of red algae waste for the production of cellulose nanocrystals and its application in polymer nanocomposites. International Journal of Biological Macromolecules 2018, 106, 681-691, https://doi.org/10.1016/j.ijbiomac.2017.08.067. 
112. Rashid, S.; Dutta, H. Characterization of nanocellulose extracted from short, medium and long grain rice husks. Industrial Crops and Products 2020, 154, https://doi.org/10.1016/j.indcrop.2020.112627.

113. Naduparambath, S.; T.V, J.; V, S.; M.P, S.; Balan, A.K.; E, P. Isolation and characterisation of cellulose nanocrystals from sago seed shells. Carbohydrate Polymers 2018, 180, 13-20, https://doi.org/10.1016/j.carbpol.2017.09.088.

114. Yang, X.; Liu, H.; Han, F.; Jiang, S.; Liu, L.; Xia, Z. Fabrication of cellulose nanocrystal from Carex meyeriana Kunth and its application in the adsorption of methylene blue. Carbohydrate Polymers 2017, 175, 464-472, https://doi.org/10.1016/j.carbpol.2017.08.007.

115. Mariano, M.; Cercená, R.; Soldi, V. Thermal characterization of cellulose nanocrystals isolated from sisal fibers using acid hydrolysis. Industrial Crops and Products 2016, 94, 454-462, https://doi.org/10.1016/j.indcrop.2016.09.011.

116. Song, K.; Zhu, X.; Zhu, W.; Li, X. Preparation and characterization of cellulose nanocrystal extracted from Calotropis procera biomass. Bioresources and Bioprocessing 2019, 6, https://doi.org/10.1186/s40643-0190279-z.

117. Flauzino Neto, W.P.; Mariano, M.; da Silva, I.S.V.; Silvério, H.A.; Putaux, J.-L.; Otaguro, H.; Pasquini, D.; Dufresne, A. Mechanical properties of natural rubber nanocomposites reinforced with high aspect ratio cellulose nanocrystals isolated from soy hulls. Carbohydrate Polymers 2016, 153, 143-152, https://doi.org/10.1016/j.carbpol.2016.07.073.

118. Kumar, A.; Negi, Y.S.; Choudhary, V.; Bhardwaj, N.K. Characterization of Cellulose Nanocrystals Produced by Acid-Hydrolysis from Sugarcane Bagasse as Agro-Waste. Journal of Materials Physics and Chemistry 2014, 2, 1-8, https://doi.org/10.12691/jmpc-2-1-1.

119. Camargo, L.A.; Pereira, S.C.; Correa, A.C.; Farinas, C.S.; Marconcini, J.M.; Mattoso, L.H.C. Feasibility of Manufacturing Cellulose Nanocrystals from the Solid Residues of Second-Generation Ethanol Production from Sugarcane Bagasse. BioEnergy Research 2016, 9, 894-906, https://doi.org/10.1007/s12155-016-97440 .

120. Evans, S.K.; Wesley, O.N.; Nathan, O.; Moloto, M.J. Chemically purified cellulose and its nanocrystals from sugarcane baggase: isolation and characterization. Heliyon 2019, 5, https://doi.org/10.1016/j.heliyon.2019.e02635.

121. Shanshan, C.; Tao, H.; Wang, Y.; Zhongsu, M.; Liping, Z. Process optimization of nanocrystalline cellulose from sunflower seed hull and its characterization. Transactions of the Chinese Society of Agricultural Engineering 2015, 31, 302-308, https://doi.org/10.11975/j.issn.1002-6819.2015.15.041.

122. Wu, Q.; Meng, Y.; Concha, K.; Wang, S.; Li, Y.; Ma, L.; Fu, S. Influence of temperature and humidity on nano-mechanical properties of cellulose nanocrystal films made from switchgrass and cotton. Industrial Crops and Products 2013, 48, 28-35, https://doi.org/10.1016/j.indcrop.2013.03.032.

123. Jiang, F.; Hsieh, Y.-L. Cellulose nanocrystal isolation from tomato peels and assembled nanofibers. Carbohydrate Polymers 2015, 122, 60-68, https://doi.org/10.1016/j.carbpol.2014.12.064.

124. Zhao, Y.; Zhang, Y.; Lindström, M.E.; Li, J. Tunicate cellulose nanocrystals: Preparation, neat films and nanocomposite films with glucomannans. Carbohydrate Polymers 2015, 117, 286-296, https://doi.org/10.1016/j.carbpol.2014.09.020.

125. El Achaby, M.; El Miri, N.; Hannache, H.; Gmouh, S.; Ben youcef, H.; Aboulkas, A. Production of cellulose nanocrystals from vine shoots and their use for the development of nanocomposite materials. International Journal of Biological Macromolecules 2018, 117, 592-600, https://doi.org/10.1016/j.ijbiomac.2018.05.201.

126. Thambiraj, S.; Ravi Shankaran, D. Preparation and physicochemical characterization of cellulose nanocrystals from industrial waste cotton. Applied Surface Science 2017, 412, 405-416, https://doi.org/10.1016/j.apsusc.2017.03.272.

127. Wang, Z.; Yao, Z.; Zhou, J.; Zhang, Y. Reuse of waste cotton cloth for the extraction of cellulose nanocrystals. Carbohydrate Polymers 2017, 157, 945-952, https://doi.org/10.1016/j.carbpol.2016.10.044.

128. Pandi, N.; Sonawane, S.H.; Anand Kishore, K. Synthesis of cellulose nanocrystals (CNCs) from cotton using ultrasound-assisted acid hydrolysis. Ultrasonics $\quad$ Sonochemistry 2021, 70, https://doi.org/10.1016/j.ultsonch.2020.105353.

129. Pandi, N.; Sonawane, S.H.; Anand Kishore, K. Synthesis of cellulose nanocrystals (CNCs) from cotton using ultrasound-assisted acid hydrolysis. Ultrasonics Sonochemistry 2021, 70, https://doi.org/10.1016/j.carbpol.2020.115997.

130. Espíndola, S.P.; Pronk, M.; Zlopasa, J.; Picken, S.J.; van Loosdrecht, M.C.M. Nanocellulose recovery from domestic wastewater. Journal of Cleaner Production 2821, https://doi.org/10.1016/j.jclepro.2020.124507.

131. Danial, W.H.; Mohd Taib, R.; Abu Samah, M.A.; Mohd Salim, R.; Abdul Majid, Z. The valorization of municipal grass waste for the extraction of cellulose nanocrystals. RSC Advances 2020, 10,https://doi.org/10.1039/D0RA07972C.

132. Campano, C.; Miranda, R.; Merayo, N.; Negro, C.; Blanco, A. Direct production of cellulose nanocrystals from old newspapers and recycled newsprint. Carbohydrate Polymers 2017, 173, 489-496, https://doi.org/10.1016/j.carbpol.2017.05.073. 
133. Majumdar, S.; Paul, I.; Dey, S.; Dutta, S.; Mandal, T.; Mandal, D.D. Biotransformation of paper mill sludge by Serratia marcescens NITDPER1 for prodigiosin and cellulose nanocrystals: A strategic valorization approach. Biochemical Engineering Journal 2020, 164, https://doi.org/10.1016/j.bej.2020.107766.

134. Aguayo, M.G.; Fernández Pérez, A.; Reyes, G.; Oviedo, C.; Gacitúa, W.; Gonzalez, R.; Uyarte, O. Isolation and Characterization of Cellulose Nanocrystals from Rejected Fibers Originated in the Kraft Pulping Process. Polymers 2018, 10, https://doi.org/10.3390/polym10101145.

135. Shaheen, T.I.; Emam, H.E. Sono-chemical synthesis of cellulose nanocrystals from wood sawdust using Acid hydrolysis. International Journal of Biological Macromolecules 2018, 107, 1599-1606, https://doi.org/10.1016/j.ijbiomac.2017.10.028.

136. Xing, L.; Gu, J.; Zhang, W.; Tu, D.; Hu, C. Cellulose I and II nanocrystals produced by sulfuric acid hydrolysis of Tetra pak cellulose I. Carbohydrate Polymers 2018, 192, 184-192, https://doi.org/10.1016/j.carbpol.2018.03.042.

137. Diop, C.I.K.; Lavoie, J.-M. Isolation of Nanocrystalline Cellulose: A Technological Route for Valorizing Recycled Tetra Pak Aseptic Multilayered Food Packaging Wastes. Waste and Biomass Valorization 2017, 8, 41-56, https://doi.org/10.1007/s12649-016-9585-2.

138. Couret, L.; Irle, M.; Belloncle, C.; Cathala, B. Extraction and characterization of cellulose nanocrystals from post-consumer wood fiberboard waste. Cellulose 2017, 24, 2125-2137, https://doi.org/10.1007/s10570-0171252-7.

139. Wang, H.; Xie, H.; Du, H.; Wang, X.; Liu, W.; Duan, Y.; Zhang, X.; Sun, L.; Zhang, X.; Si, C. Highly Efficient Preparation of Functional and Thermostable Cellulose Nanocrystals via H2SO4 Intensified Acetic Acid Hydrolysis. Carbohydrate Polymers 2020, 239, https://doi.org/10.1016/j.carbpol.2020.116233.

140. Bao, C.; Chen, X.; Liu, C.; Liao, Y.; Huang, Y.; Hao, L.; Yan, H.; Lin, Q. Extraction of cellulose nanocrystals from microcrystalline cellulose for the stabilization of cetyltrimethylammonium bromide-enhanced Pickering emulsions. Colloids and Surfaces A: Physicochemical and Engineering Aspects 2021, 608, https://doi.org/10.1016/j.colsurfa.2020.125442. 